ISSN-PRINT

1794-9831

E-ISSN 2322-7028

Vol. 14 No. 2

Jul - Dic 2017

Cúcuta, Colombia

Recibido:

30 de noviembre de 2016

Aprobado:

3 Mayo de

2017

* Enfermero. Magister en Epidemiología Estudiante de

Doctorado en

Enfermería.

Correo: jhon.

osorio@upb.

edu.co. Orcid:

0000-0001-6272

722 Docente.

Universidad

Pontificia

Bolivariana.

Medellín,

Colombia.

\section{La salud sexual y reproductiva como área para desarrollar la enfermería de práctica avanzada}

Sexual and reproductive health as area to develop advanced practice nursing

A saúde sexual e reprodutiva como área para desenvolver a enfermagem de prática avançada

\author{
Jhon Henry Osorio-Castaño*
}

\section{Para citar este artículo / To reference this article / Para citar este artigo/}

Osorio-Castaño JH. La salud sexual y reproductiva como área para desarrollar la enfermería de práctica avanzada. Rev. cienc. cuidad. 2017; 14(2): 130-143.

\section{Resumen}

Introducción: La enfermería de práctica avanzada implica el desarrollo de nuevos roles en la formación y práctica enfermera a partir del nivel de maestría, cuya esencia es la ampliación de los dominios de actuación con autonomía e independencia. Metodología: Revisión temática. Se realizó búsqueda en bases de datos Lilacs, Pubmed y EBSCO. Se utilizó como palabra clave enfermería de práctica avanzada, se limitó a los artículos publicados desde el año 2010 hasta el año 2016 en idioma inglés, español y portugués. Análisis: Resultados: La ley 266 de 1996 define la enfermería como como una profesión liberal. De otro lado, varias políticas, planes y programas en salud sexual y reproductiva manifiestan la necesidad de personal formado en estas temáticas y en componentes adicionales como la investigación y el liderazgo. Una oportunidad para el desarrollo de la autonomía y la independencia en enfermería es la práctica avanzada que favorezca la ampliación de los dominios de desempeño profesional y ayude a visibilizar el quehacer enfermero. Conclusión: La salud sexual y reproductiva puede ser un nicho propicio para el desarrollo de la enfermería de práctica avanzada en el marco de la atención primaria en salud siempre y cuando exista un marco político y regulatorio.

Palabras clave: Atención primaria de salud, autonomía profesional, decreto ley.

\title{
Abstract
}

Introduction: Advanced nursing practice implies the development of new roles in the nursing education and practice from a level of mastering whose essence is the extension of domains of action with autonomy and independence. Methodology: Thematic review. Research through databases Lilacs, Pubmed and EBSCO. Advanced nursing training was used as a keyword, search results were limited to the published articles from 2010 to 2016 in English, Spanish and Portuguese. Analysis: Results: The law 266 of 1996 defines nursing as a liberal profession. Moreover, various policies, plans and programs of sexual and reproductive health, express the necessity of personnel trained in these topics and in additional components such as research and leadership. An opportunity for the development of autonomy and independence in nursing is the advanced practice that promotes the extension of domains of professional performance and helps to make the nursing duties visible. Conclusion: Sexual and reproductive health may be a favorable niche for the development of advanced nursing training in the framework of primary health care as long as a policy and regulatory framework exists.

Keywords: Primary health care, professional autonomy, law decree. 
Introdução: A enfermagem de prática avançada implica o desenvolvimento de novos papeis na formação e prática de enfermagem a partir do nível de mestrado cuja essência é a ampliação dos domínios de atuação com autonomia e independência. Metodologia: Revisão temática. Realizou-se busca em bases de dados Lilacs, Pubmed e EBSCO. Utilizou-se como palavra-chave enfermagem de prática avançada, limitou-se aos artigos publicados desde o ano 2010 até o ano 2016 em idioma inglês, espanhol e português. Análise: Resultados: A lei 266 de 1996 define a enfermagem como uma profissão liberal. De outro lado, várias políticas, planos e programas em saúde sexual e reprodutiva manifestam a necessidade de pessoal formado nestas temáticas e em componentes adicionais como a pesquisa e a liderança. Uma oportunidade para o desenvolvimento da autonomia e da independência na enfermagem é a prática avançada, a qual favoreçe a ampliação dos domínios de desempenho professional e ajuda a visibilizar o trabalho do enfermeiro. Conclusão: A saúde sexual e reprodutiva pode ser um nicho adequado para o desenvolvimento da enfermagem de prática avançada no marco dos cuidados de saúde primários, sempre e quando exista um marco político e regulatório.

Palavras-chave: Autonomia professional, cuidados de saúde primários, decreto lei.

\section{Introducción}

Enfermería es una disciplina práctica que ha venido consolidando su cuerpo de conocimientos a través de modelos conceptuales, teorías de mediano rango, micro-teorías, investigación y la construcción de lenguajes estandarizados que han permitido diagnosticar, intervenir y evaluar el cuidado. De esta forma ha configurado su quehacer menos difusamente y más desligada del paraguas médico. Particularmente, la investigación se constituye en un navío colosal para enfermería, ya que es por este medio que se logra delimitar el objeto de conocimiento y evidenciar qué se hace, cómo se hace, por qué se hace y para qué se hace.

Como disciplina en crecimiento y consolidación, la enfermería ha buscado, como otras profesiones, la autonomía para ejercer sus diferentes roles en una sociedad donde su papel está más que justificado.

Esta autonomía viene dada por la capacidad y posibilidad de diagnosticar, planear, ejecutar y evaluar actividades de forma independiente y no, como expresa Camargo (1), bajo la dependencia médica y que, adicionalmente, tiene como resultado la toma de decisiones acerca del manejo y conductas a seguir en el cuidado de un usuario, familia o colectivos.

Por su parte, la independencia ha sido influenciada por varios elementos entre los que se cuentan desde la investigación a través de la formulación y ejecución de proyectos hasta la aplicación de los resultados de la misma a la práctica, el uso de teorías de enfermería en los procesos de formación, investigación y asistencia

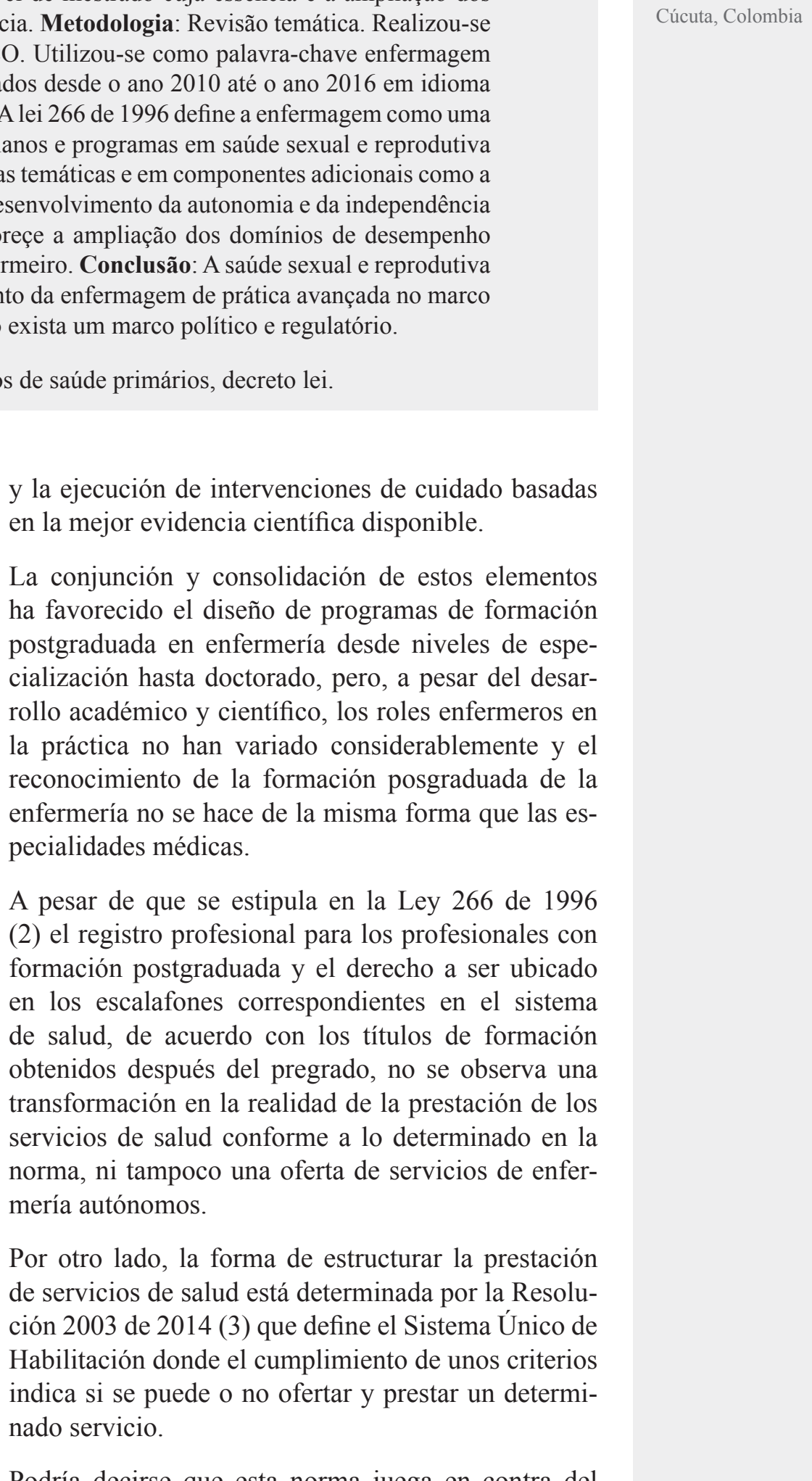

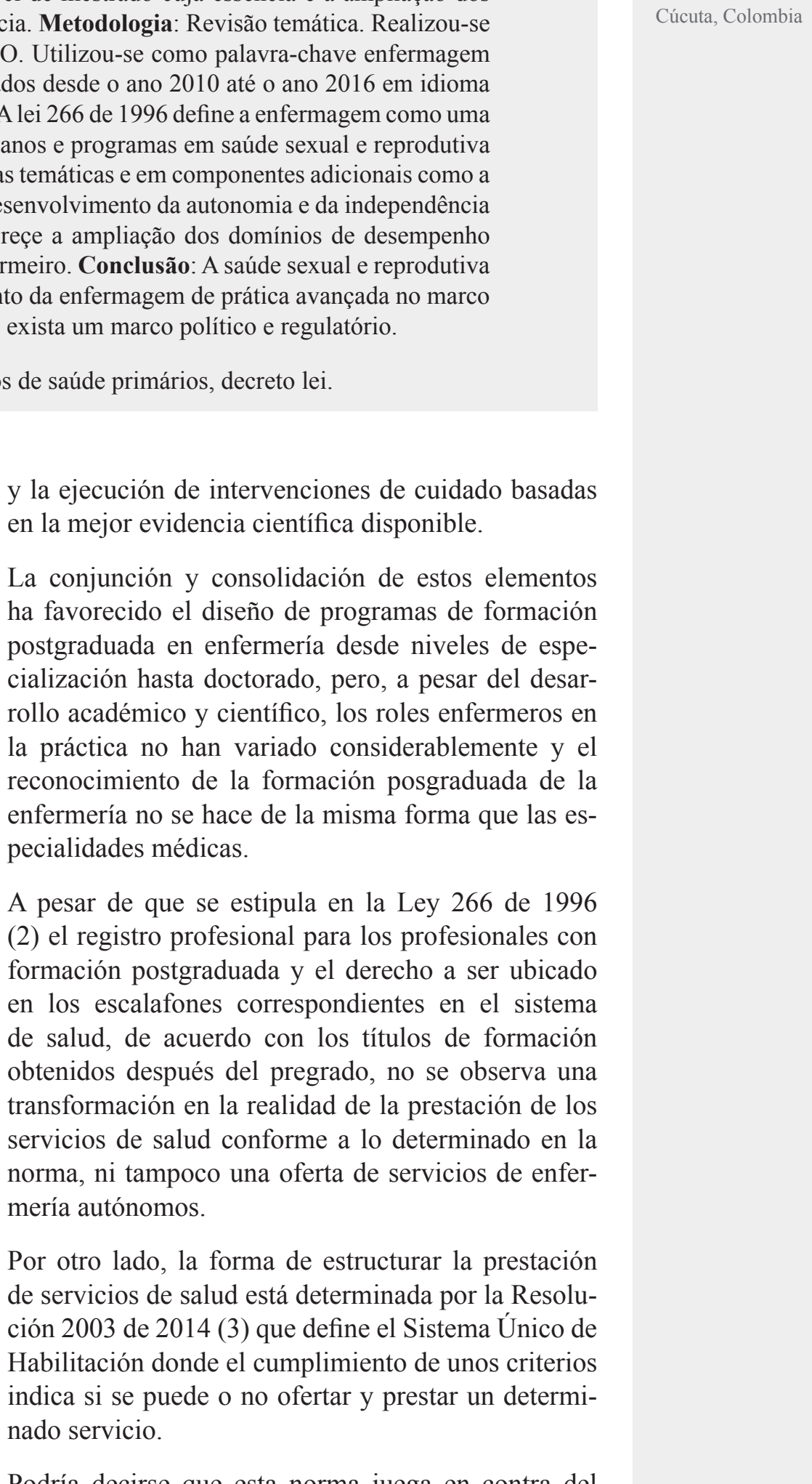

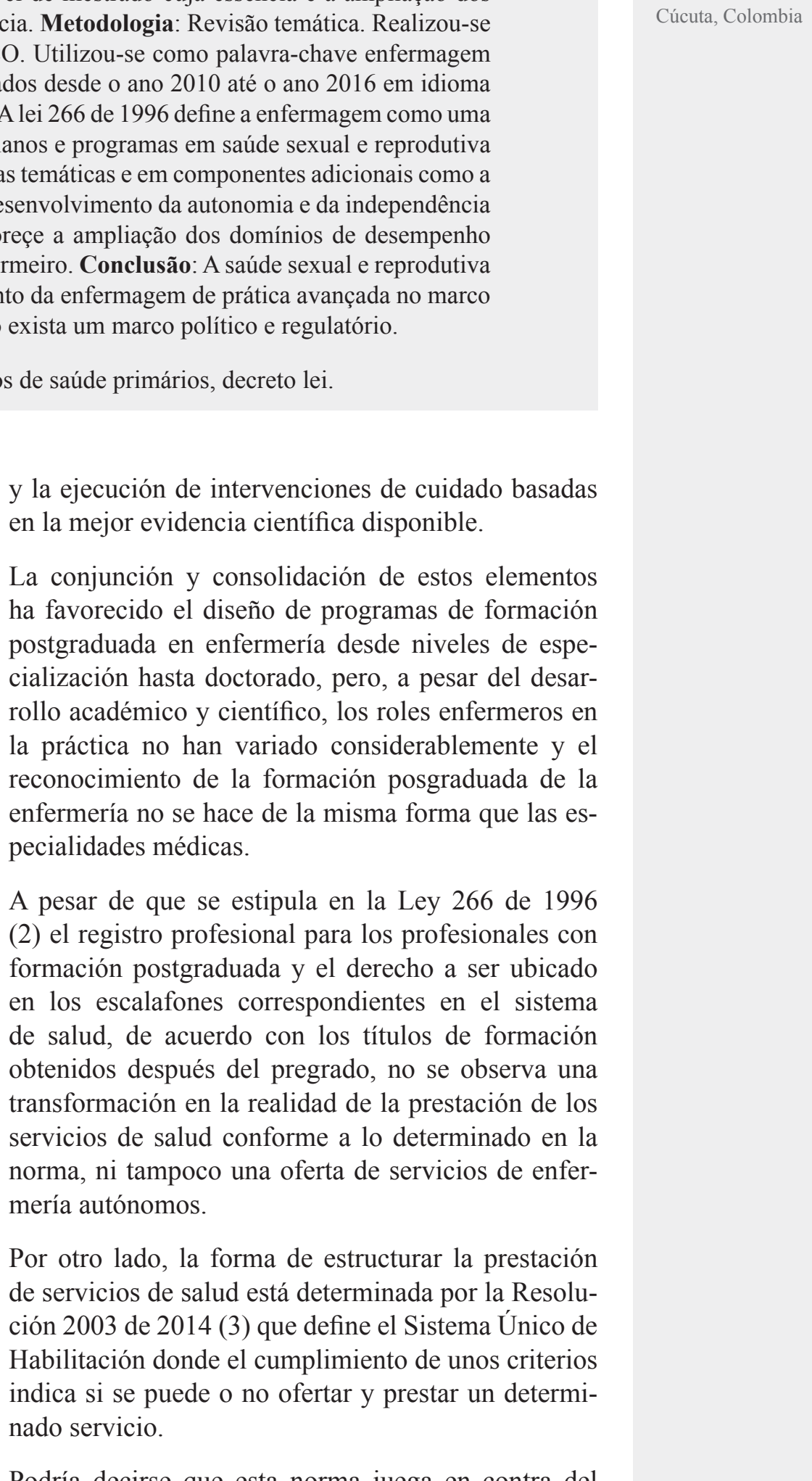

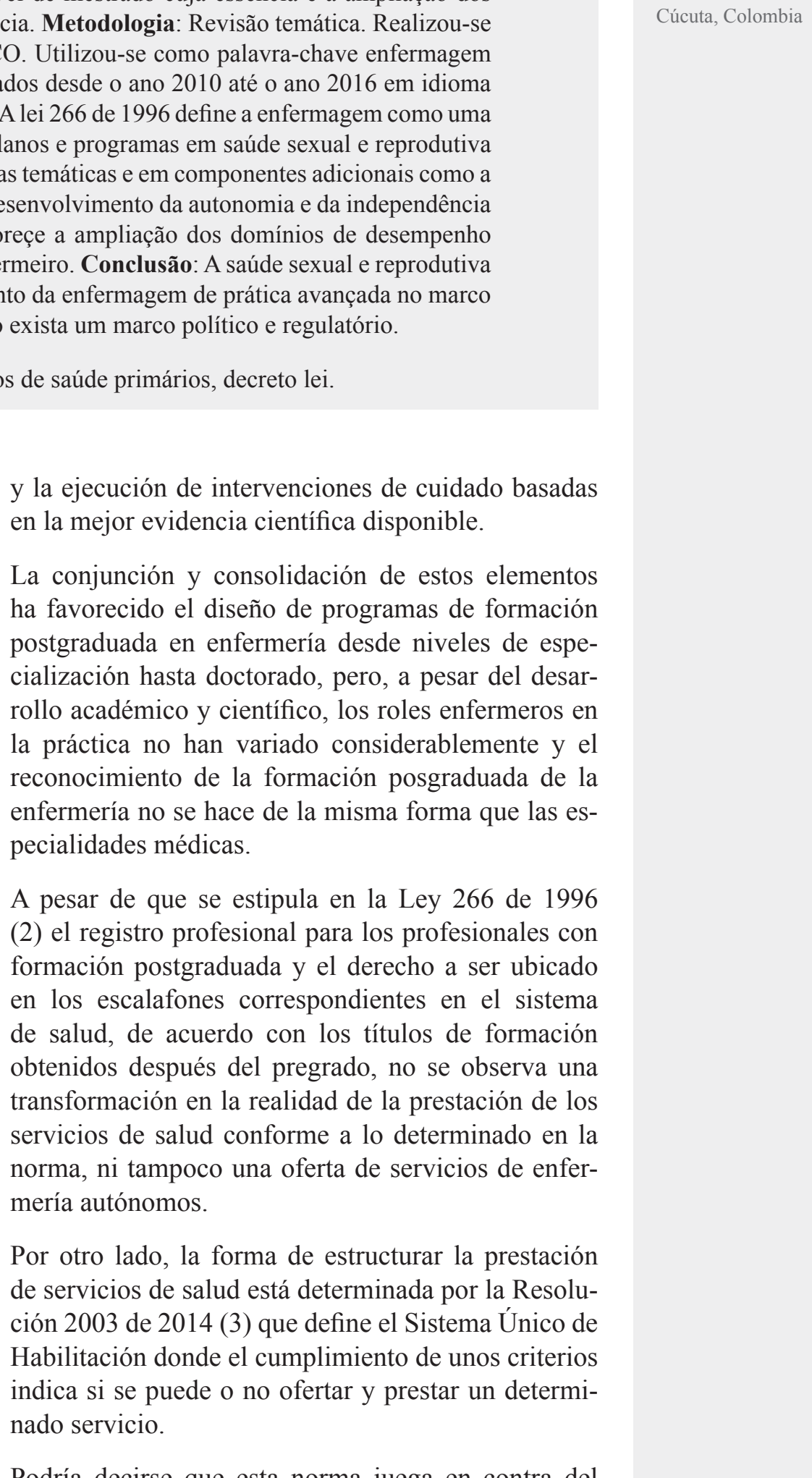

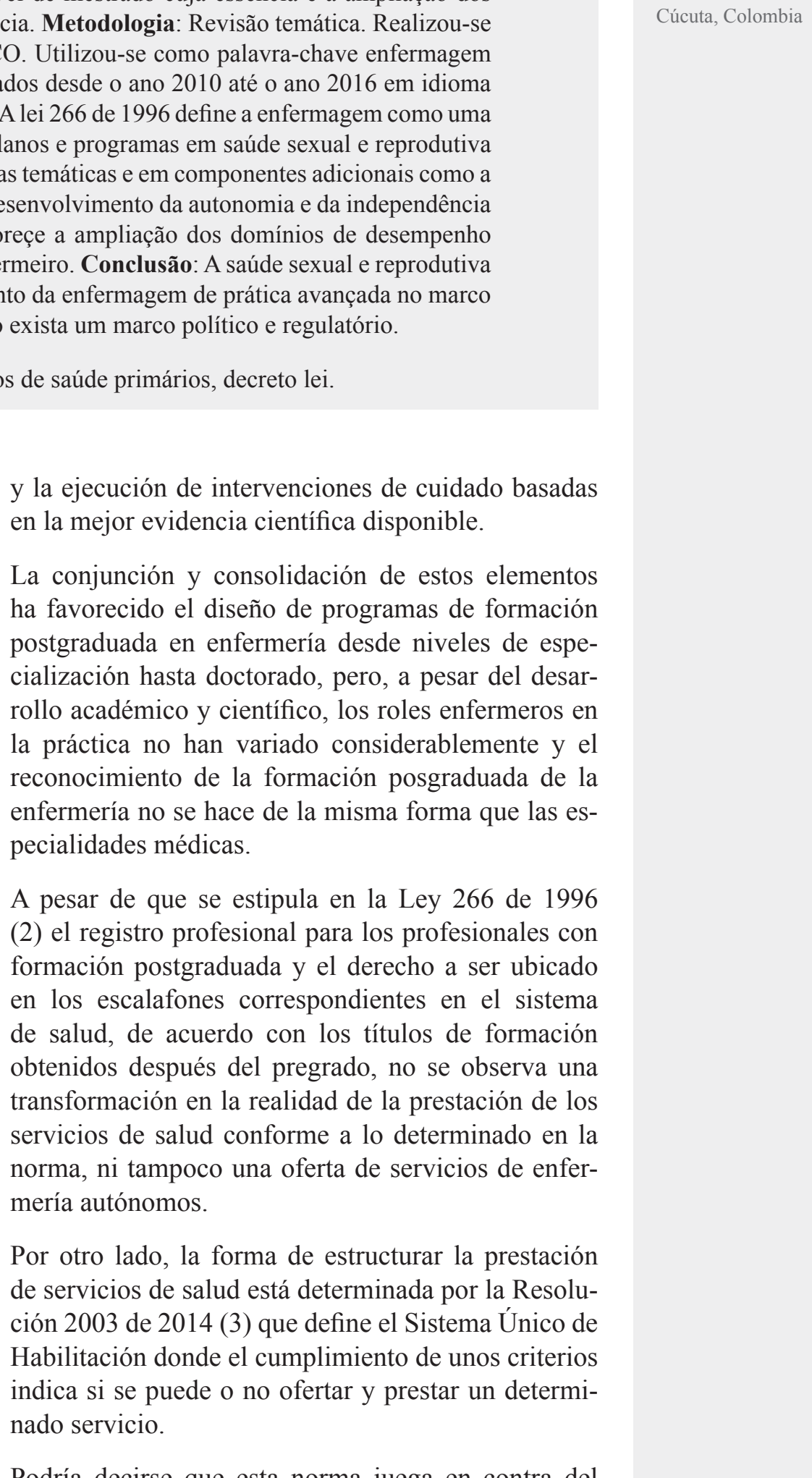

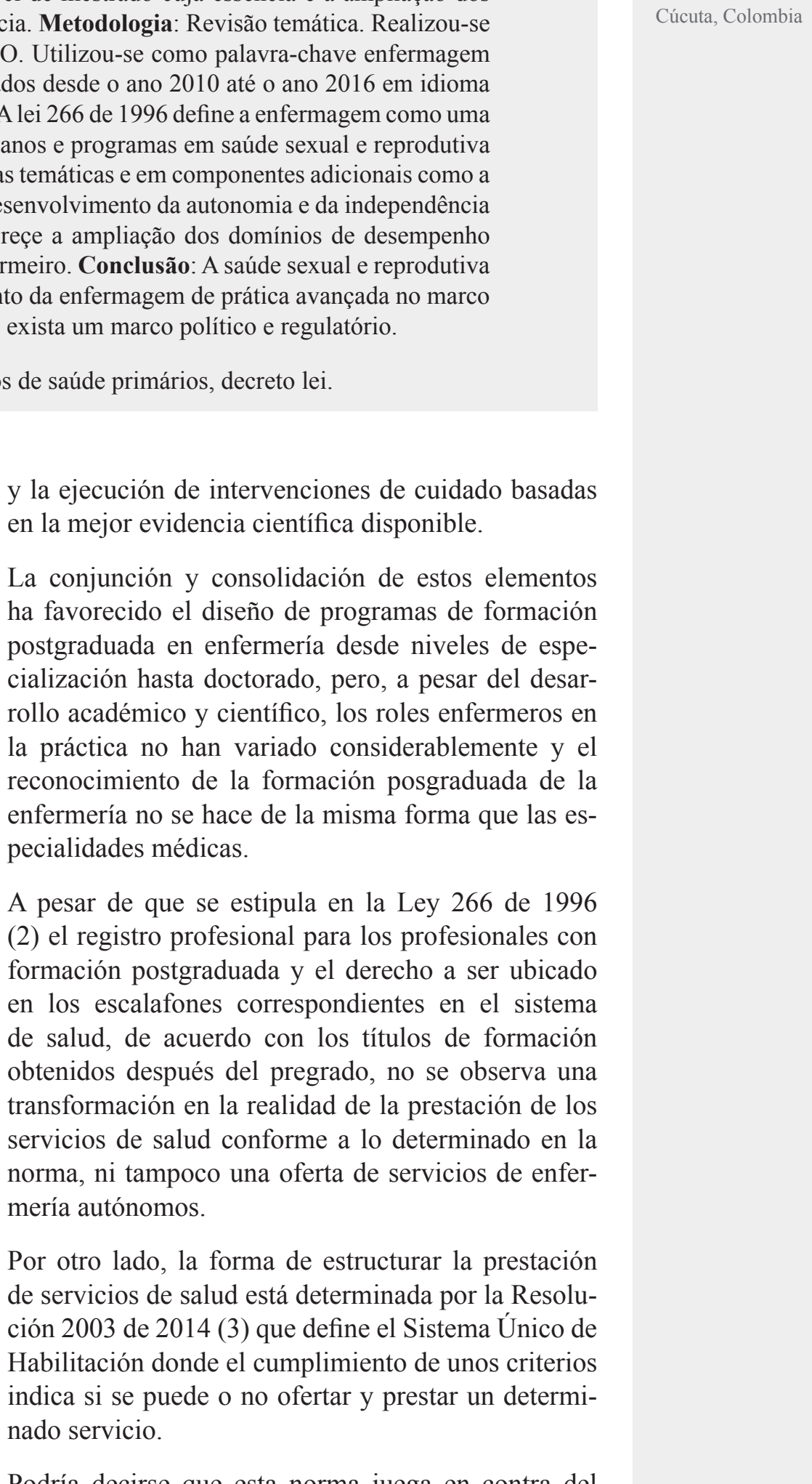

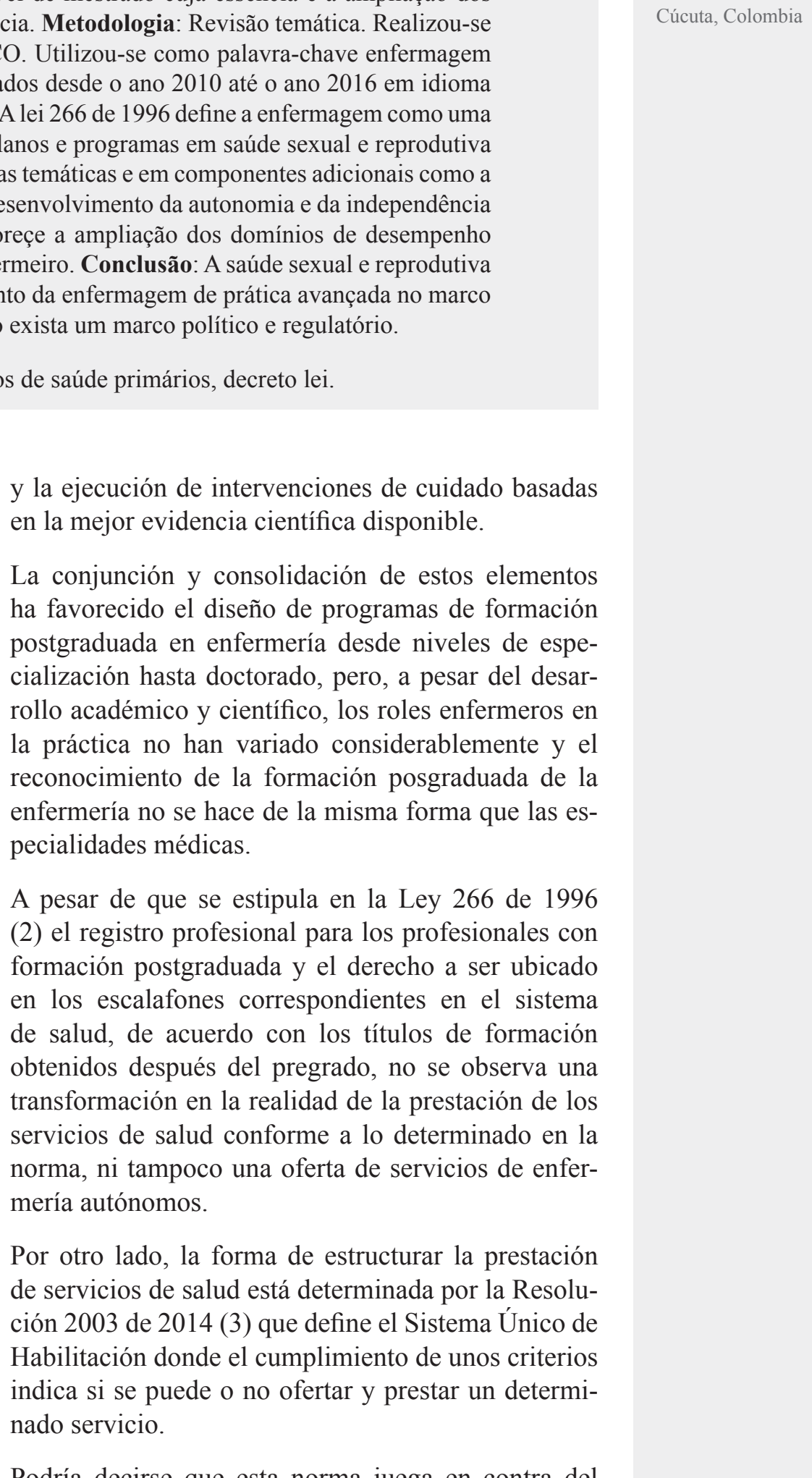

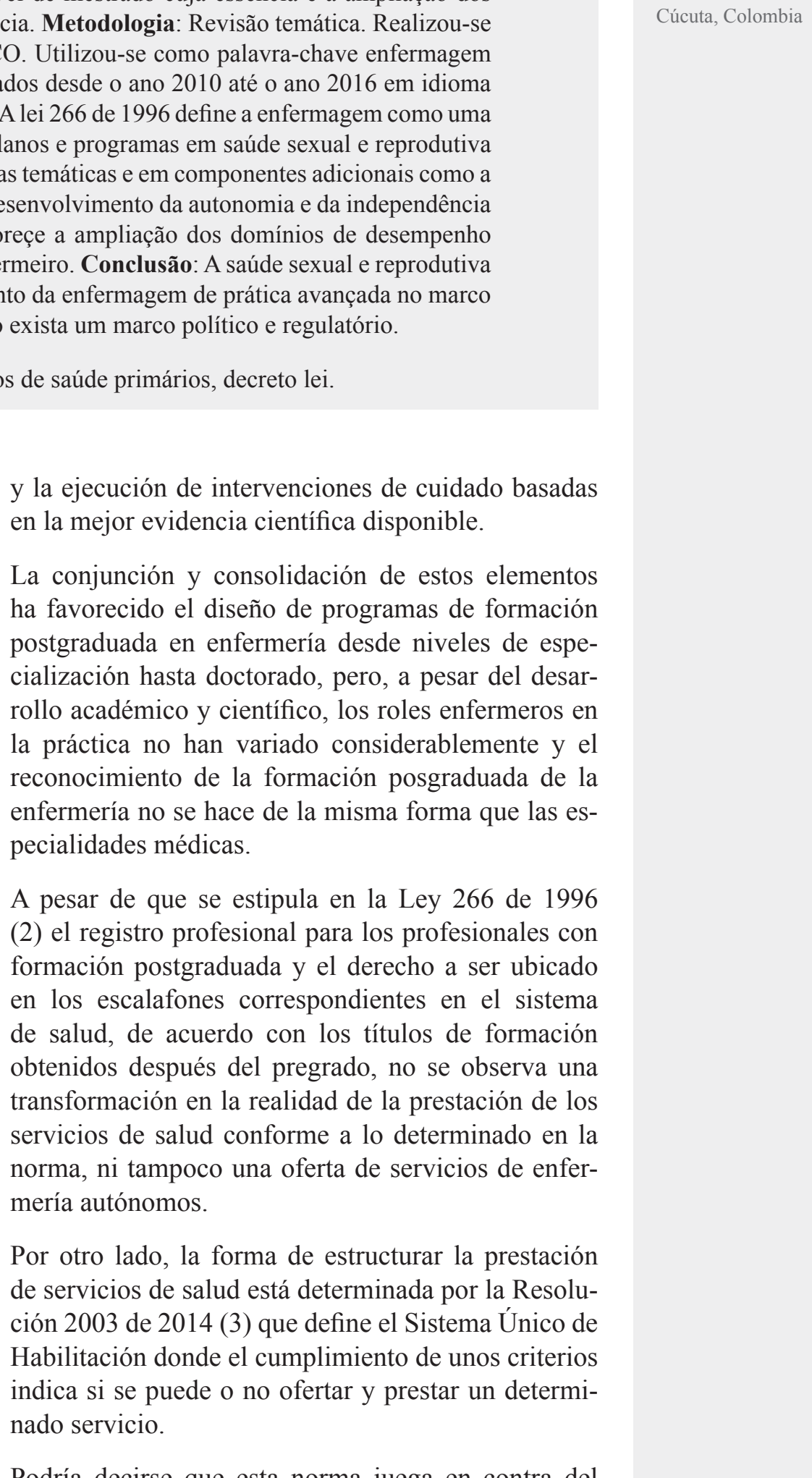

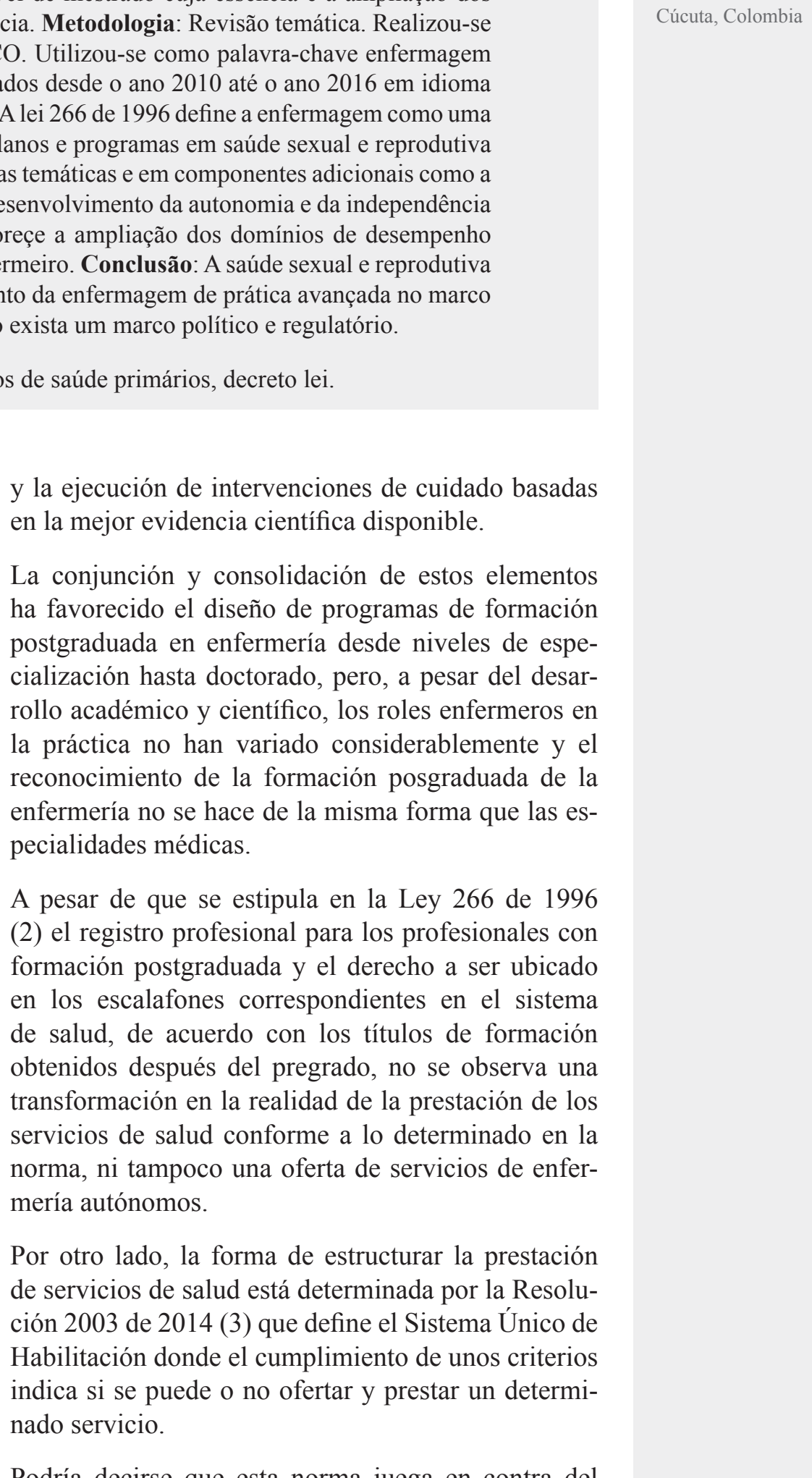

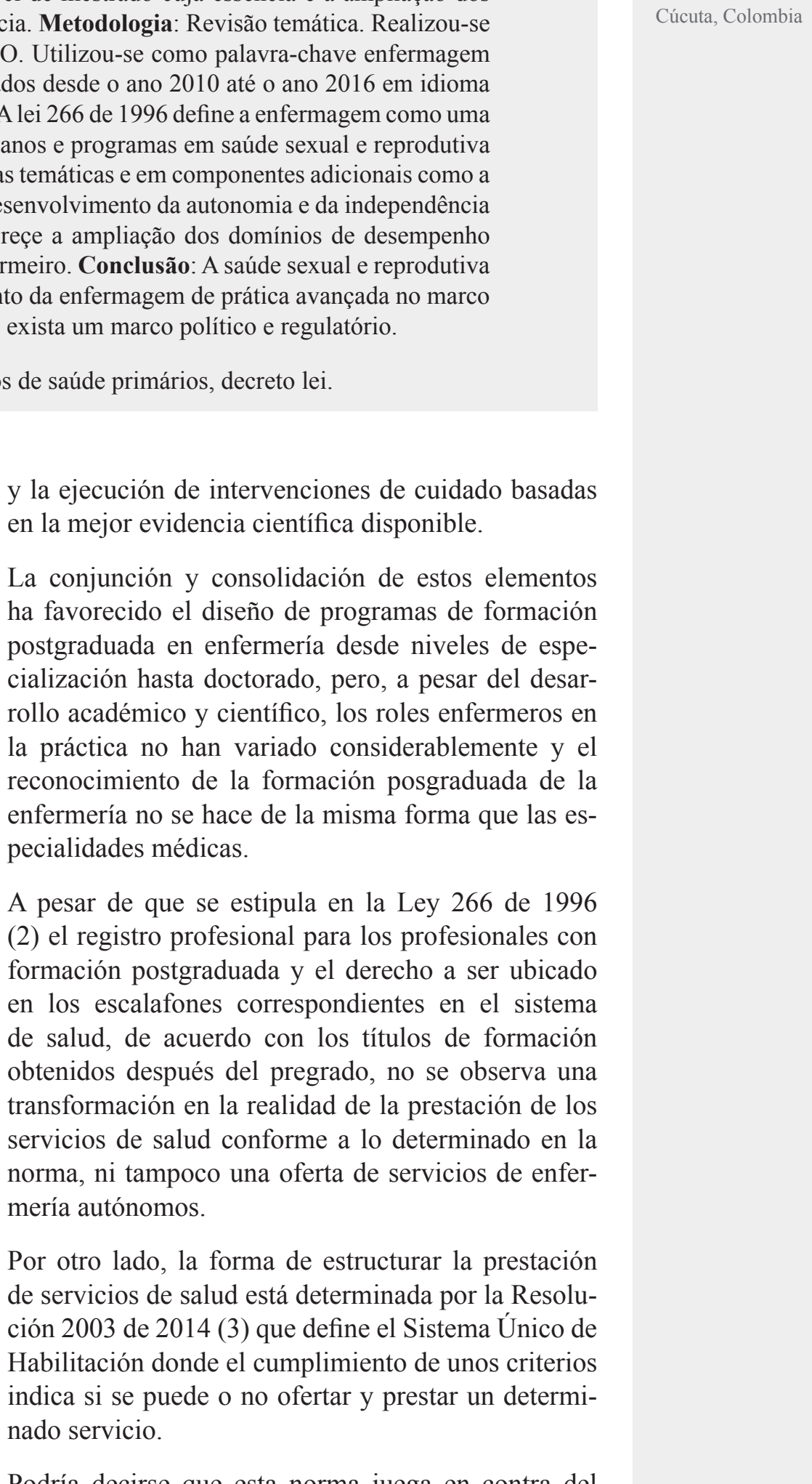

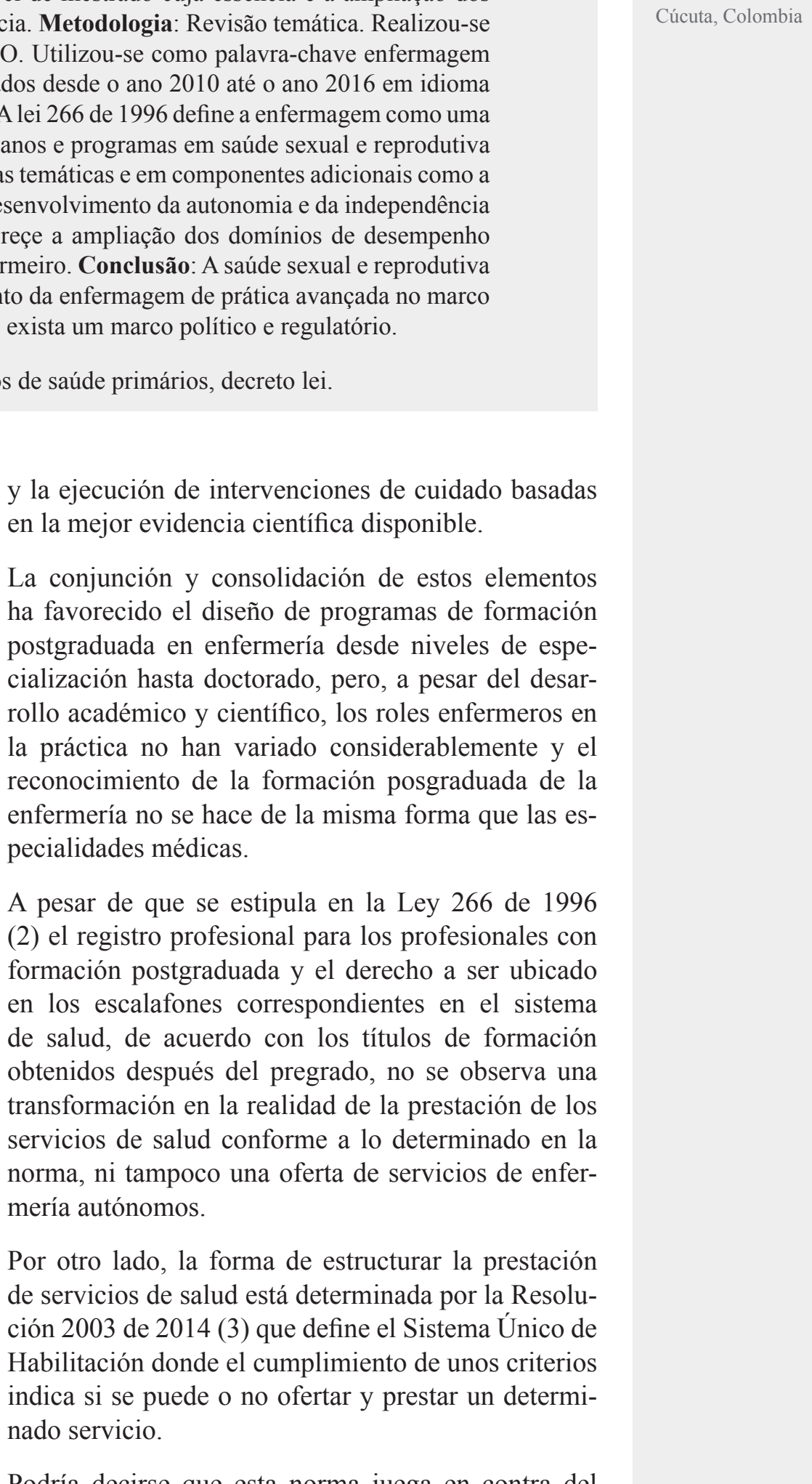

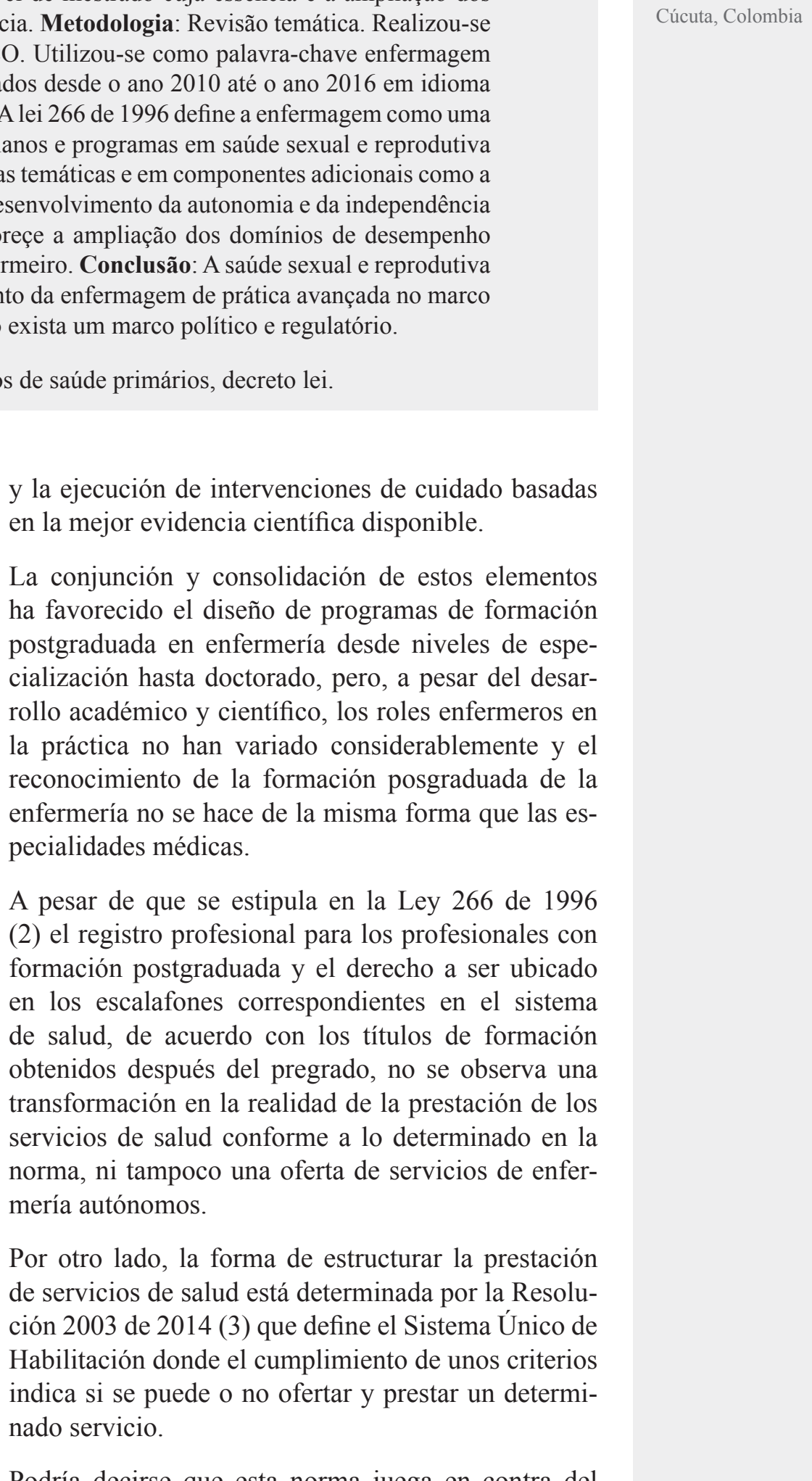

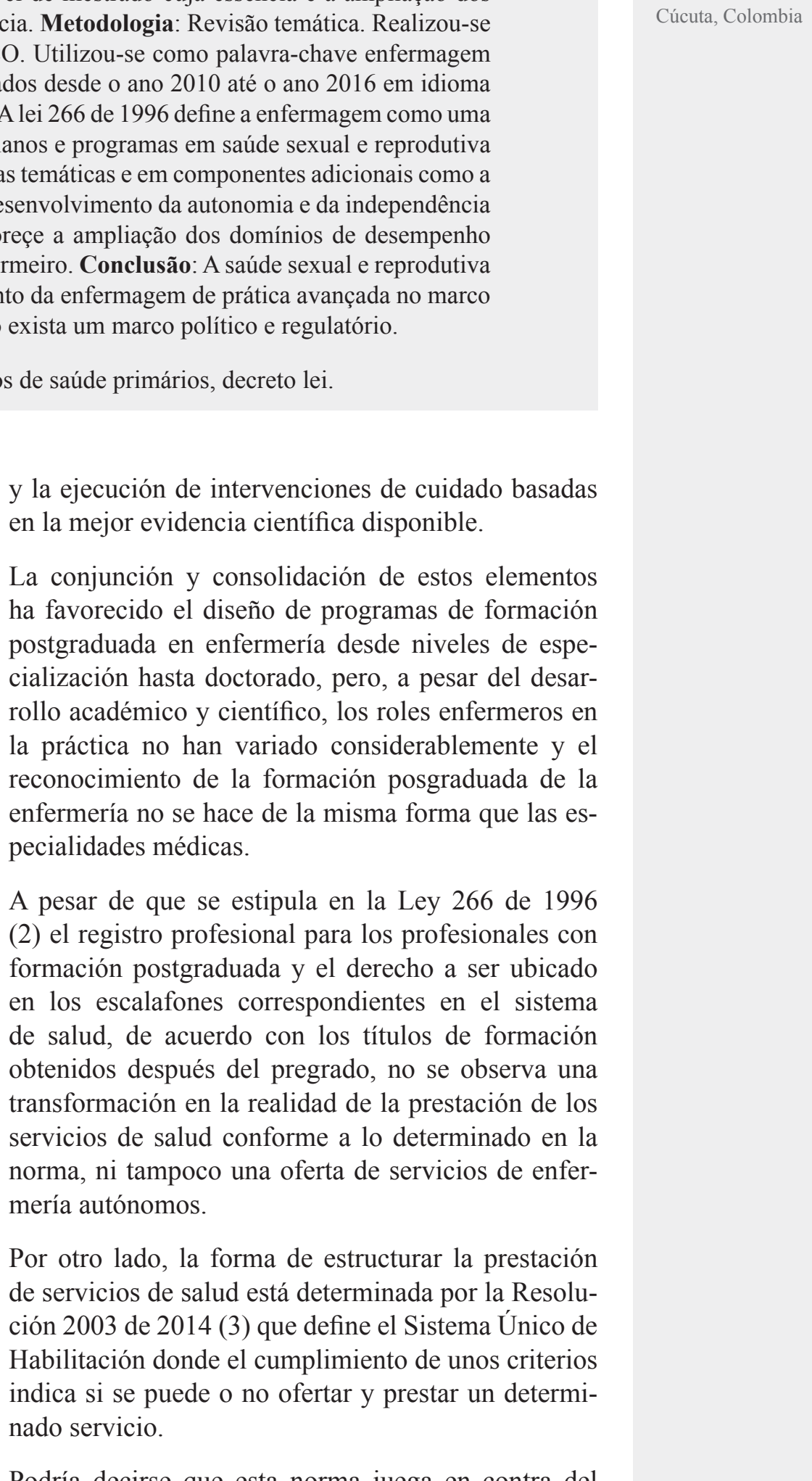

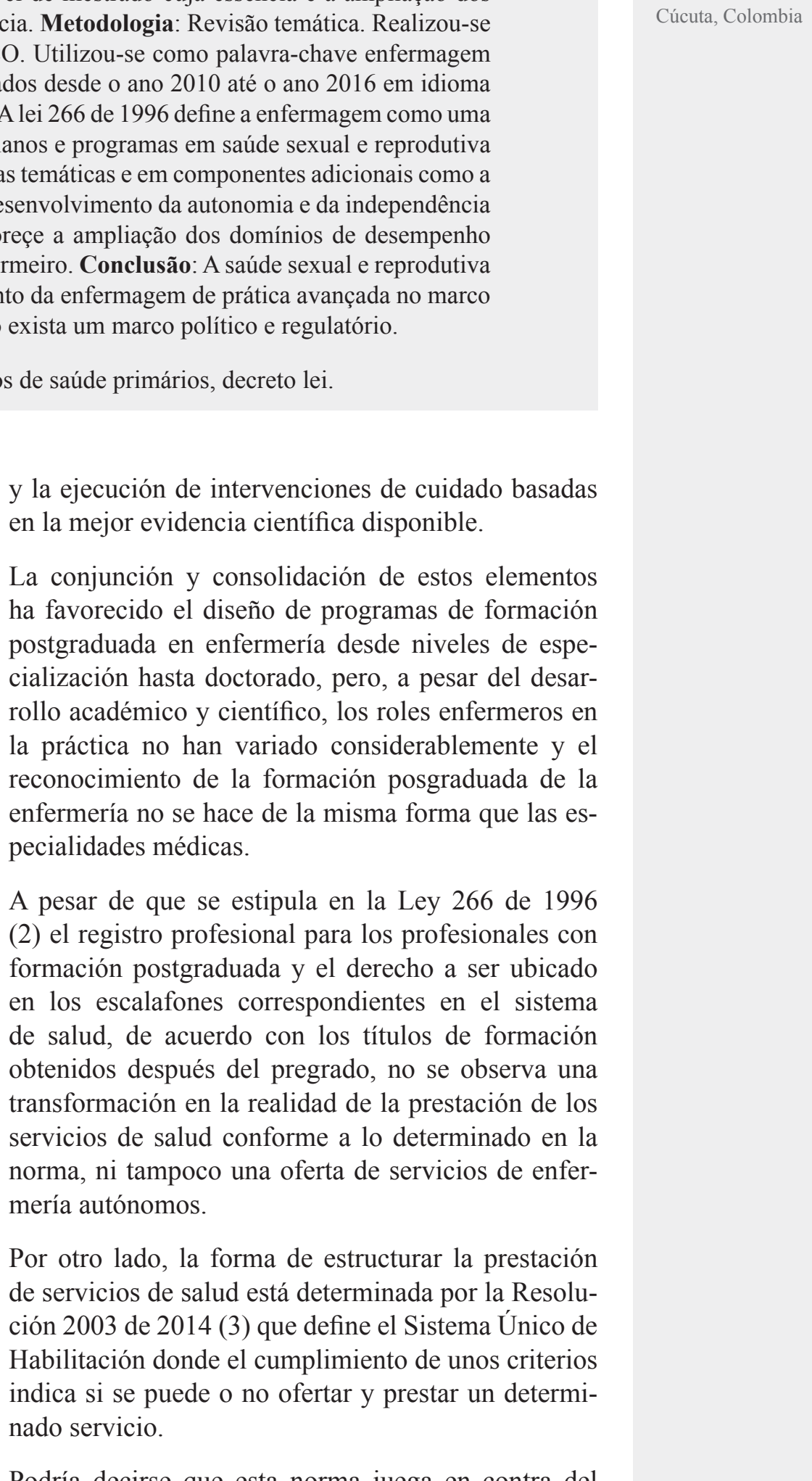

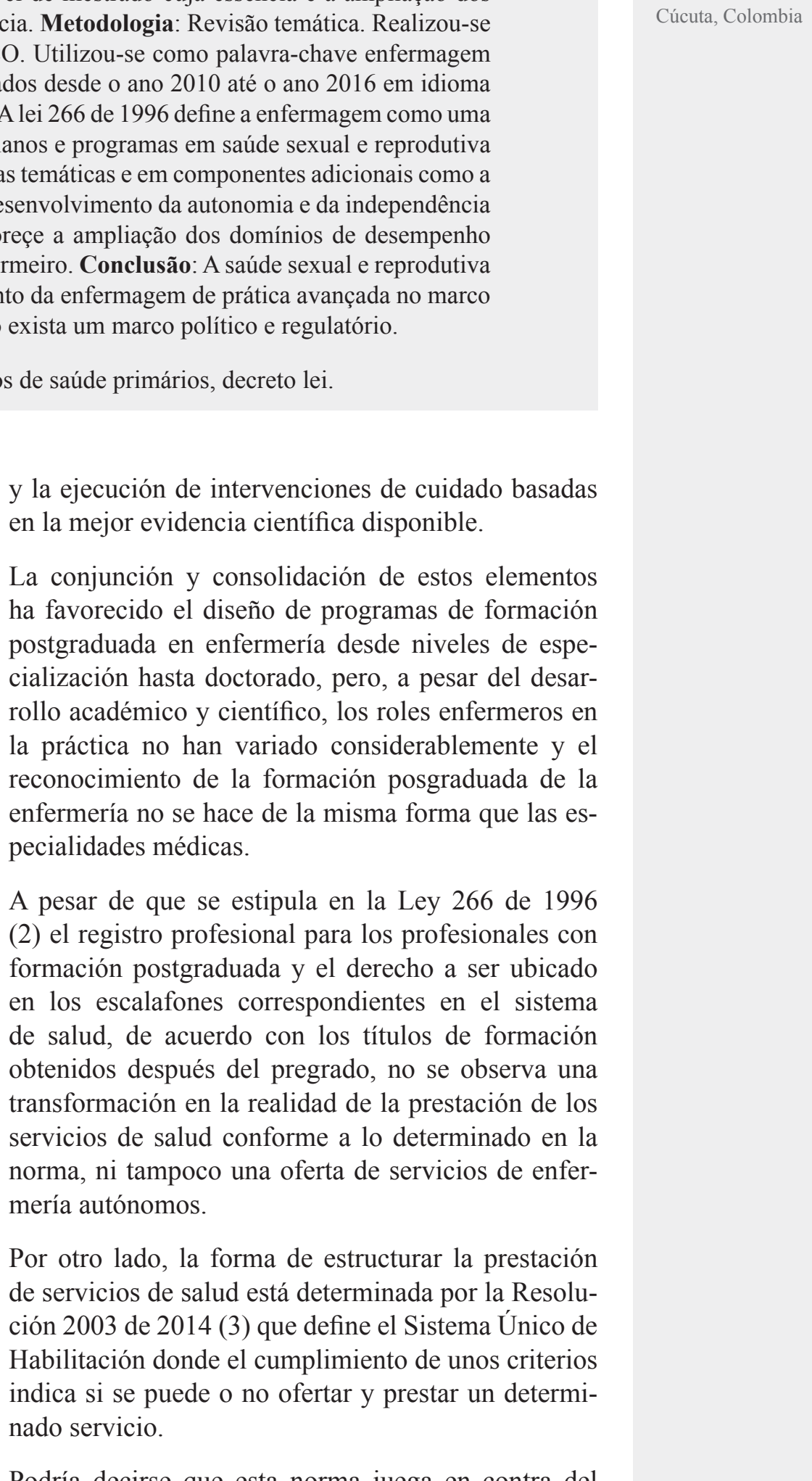

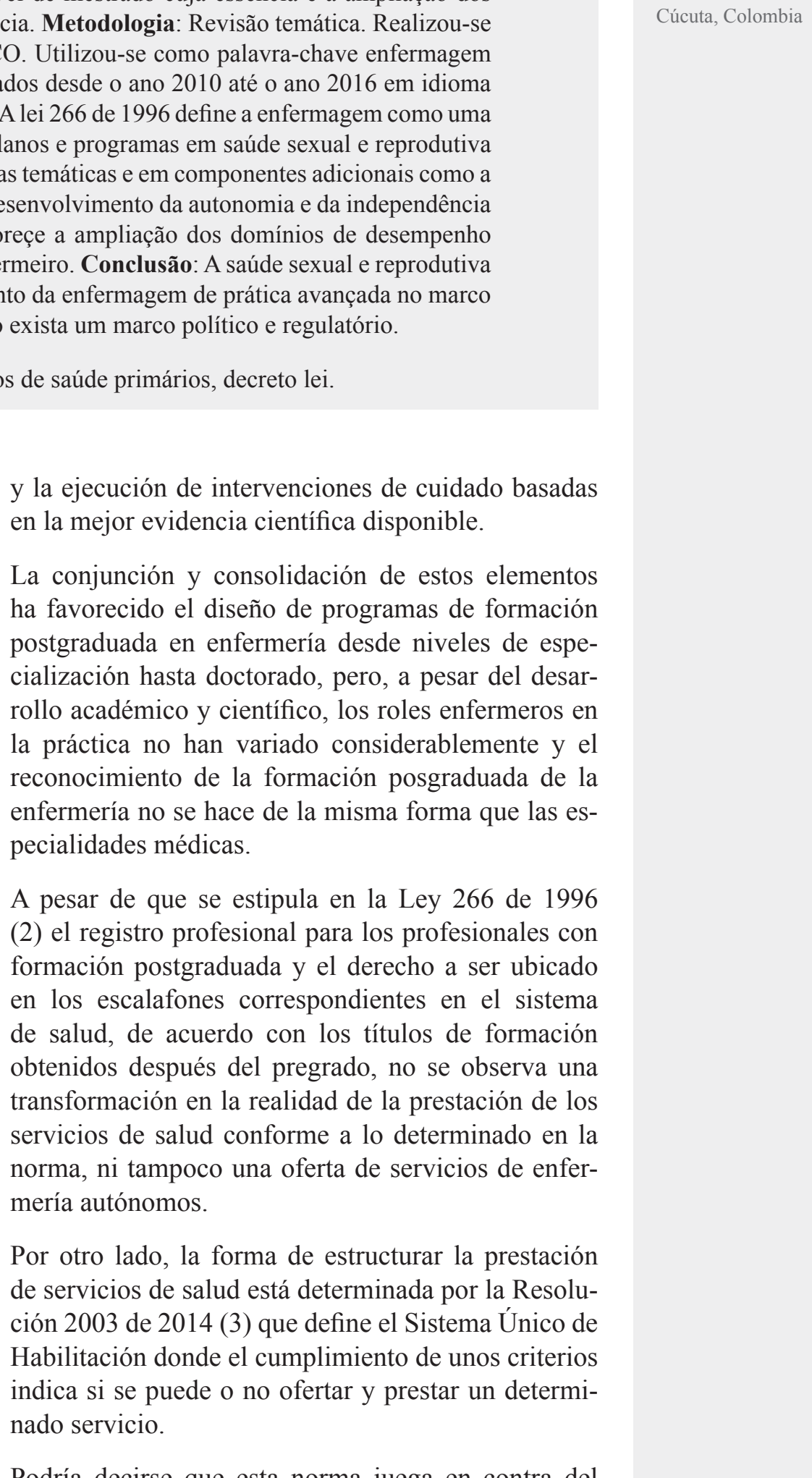

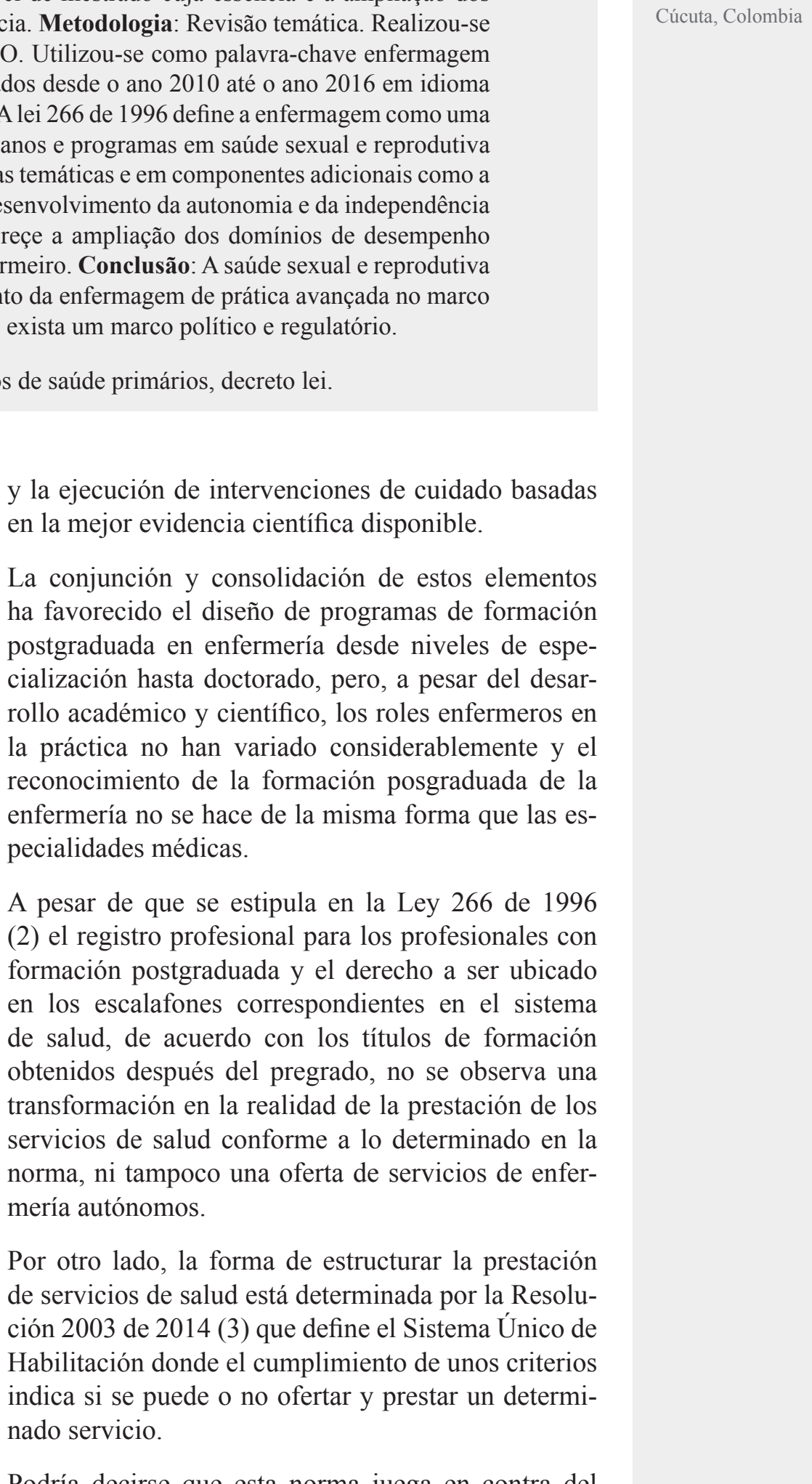

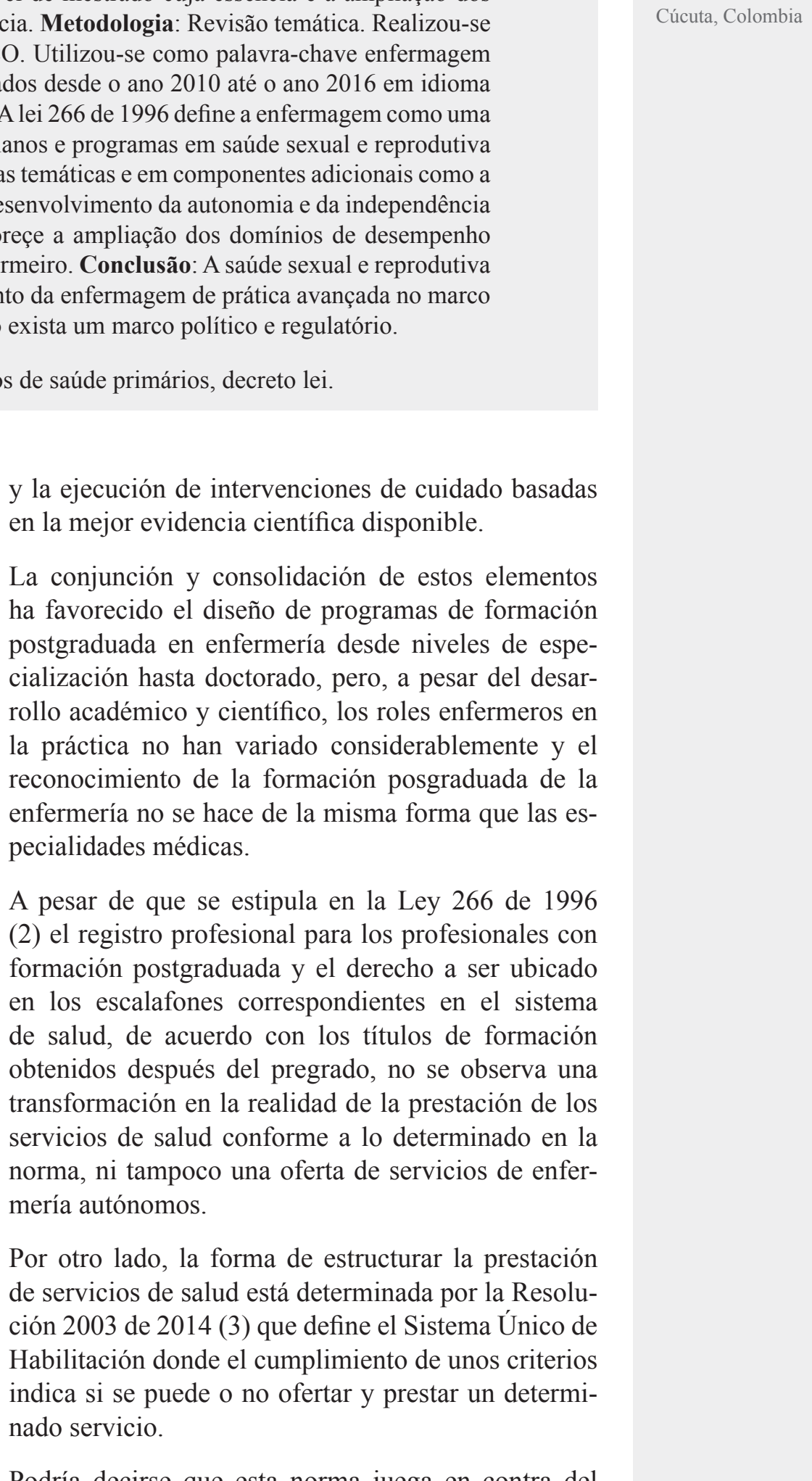

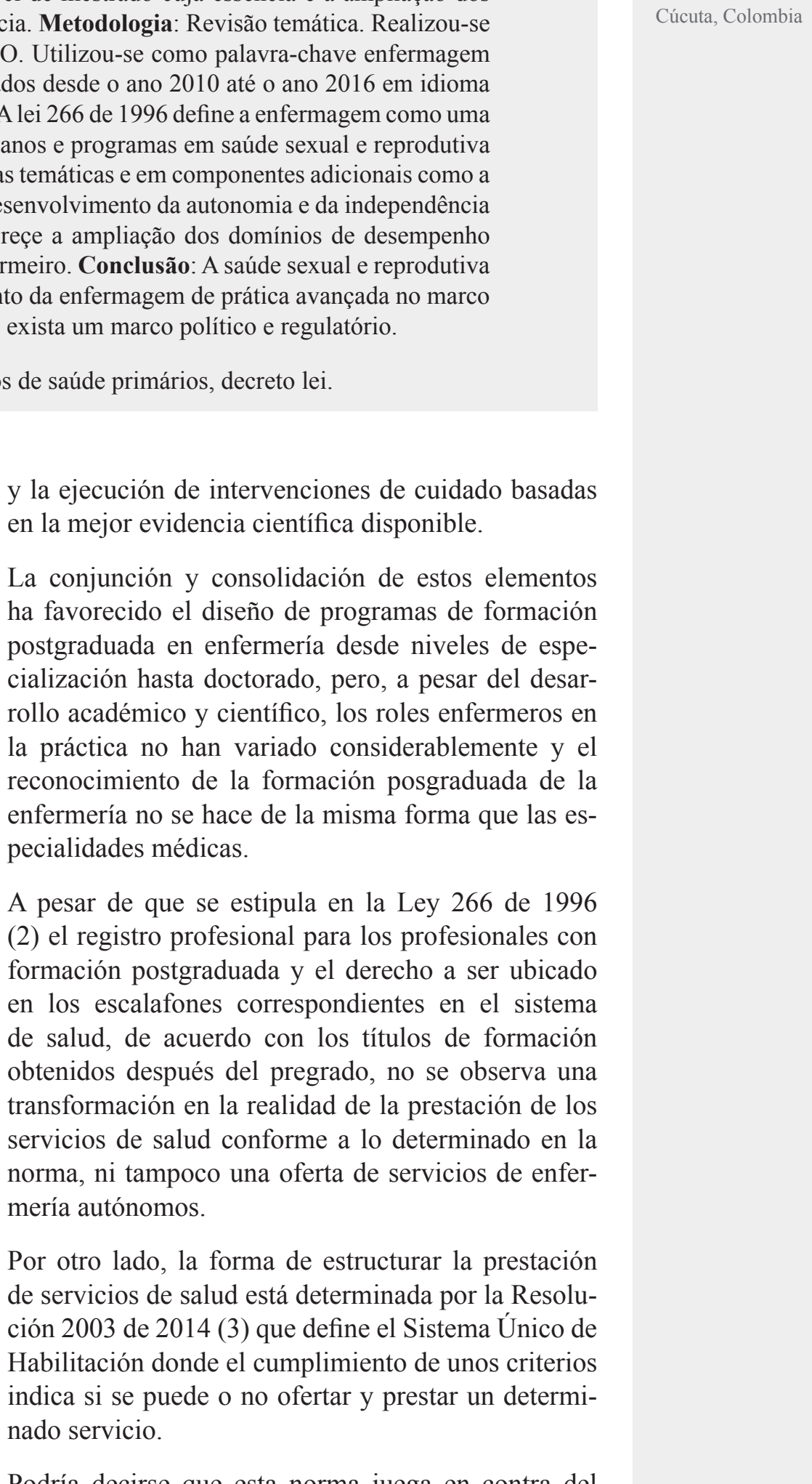

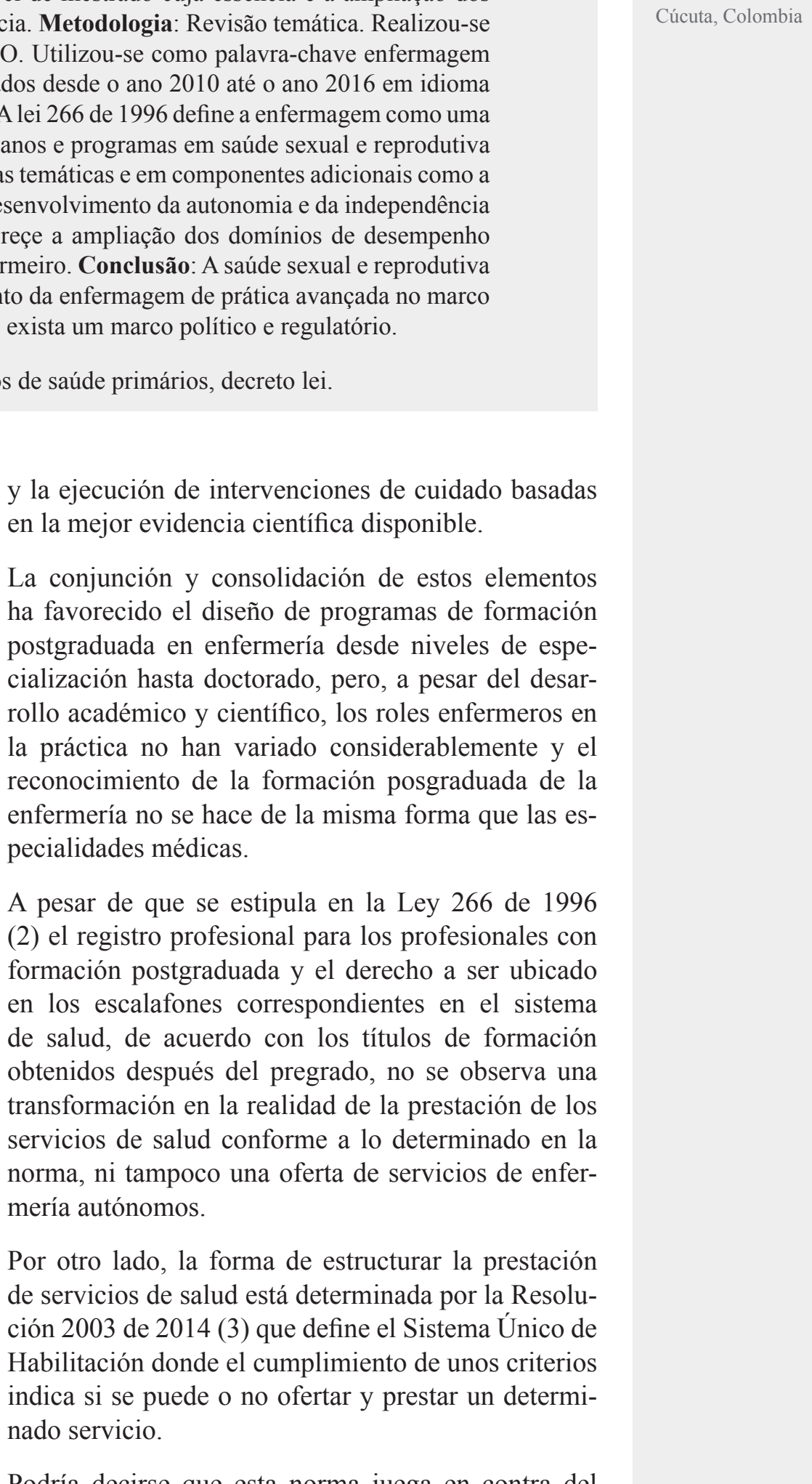

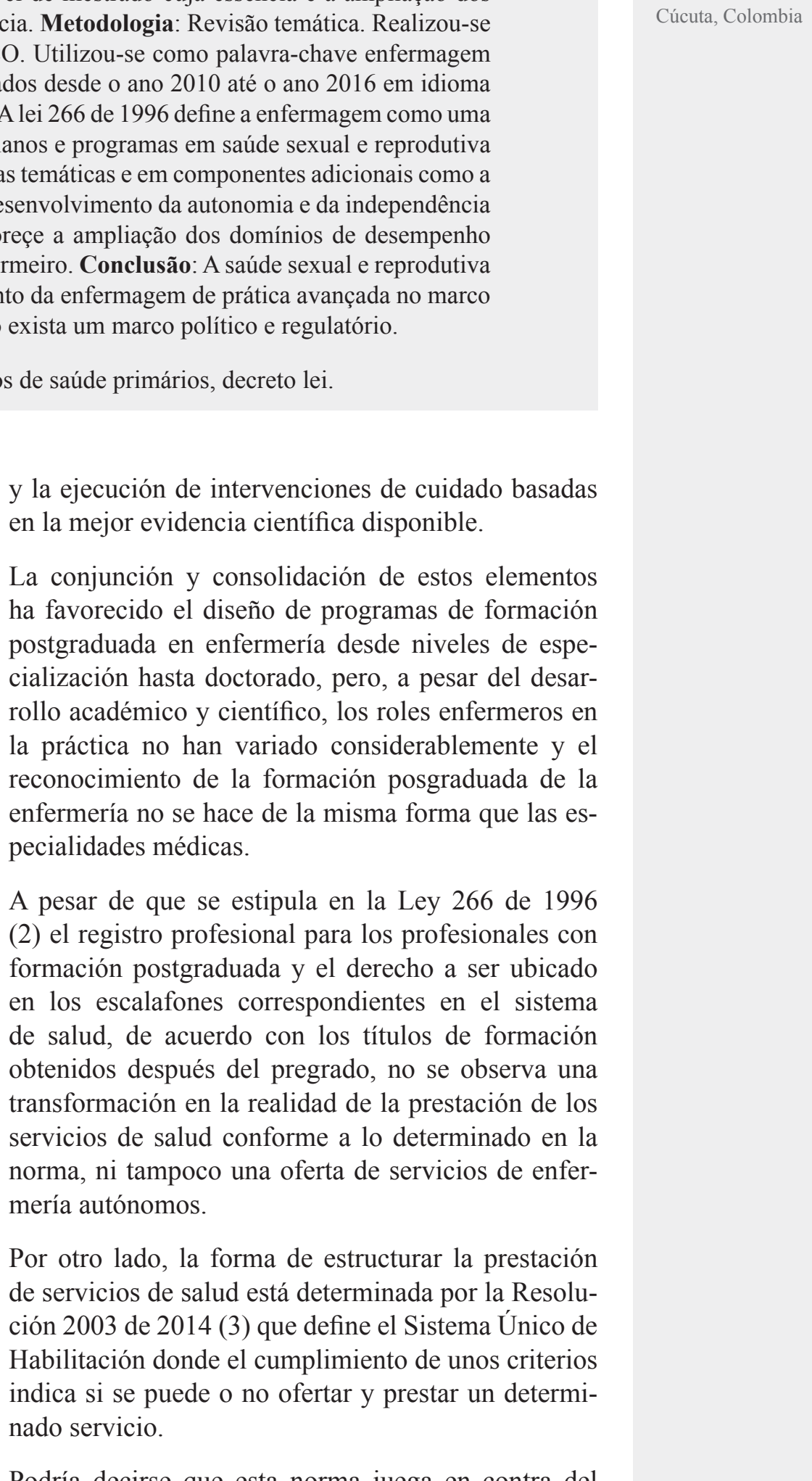

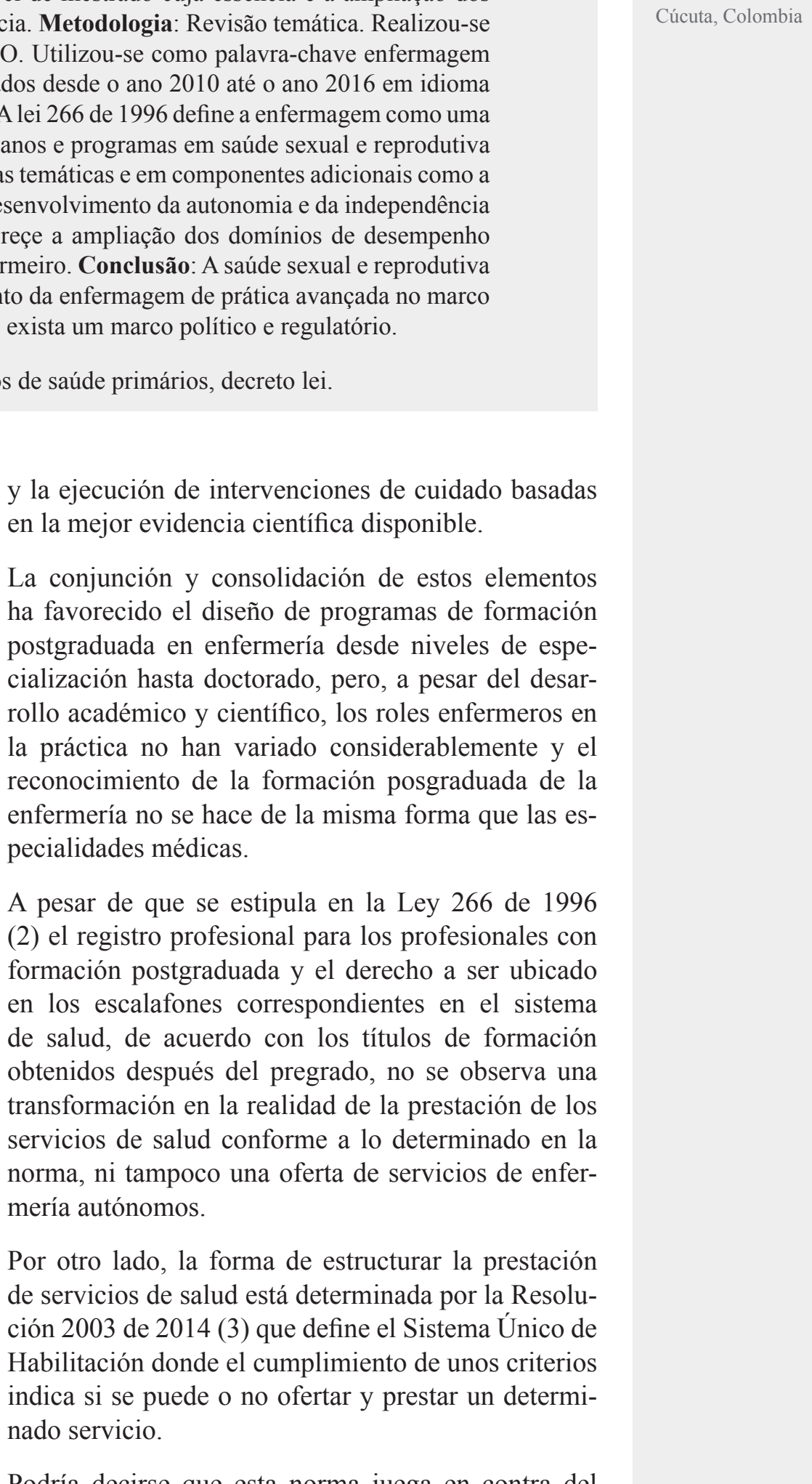

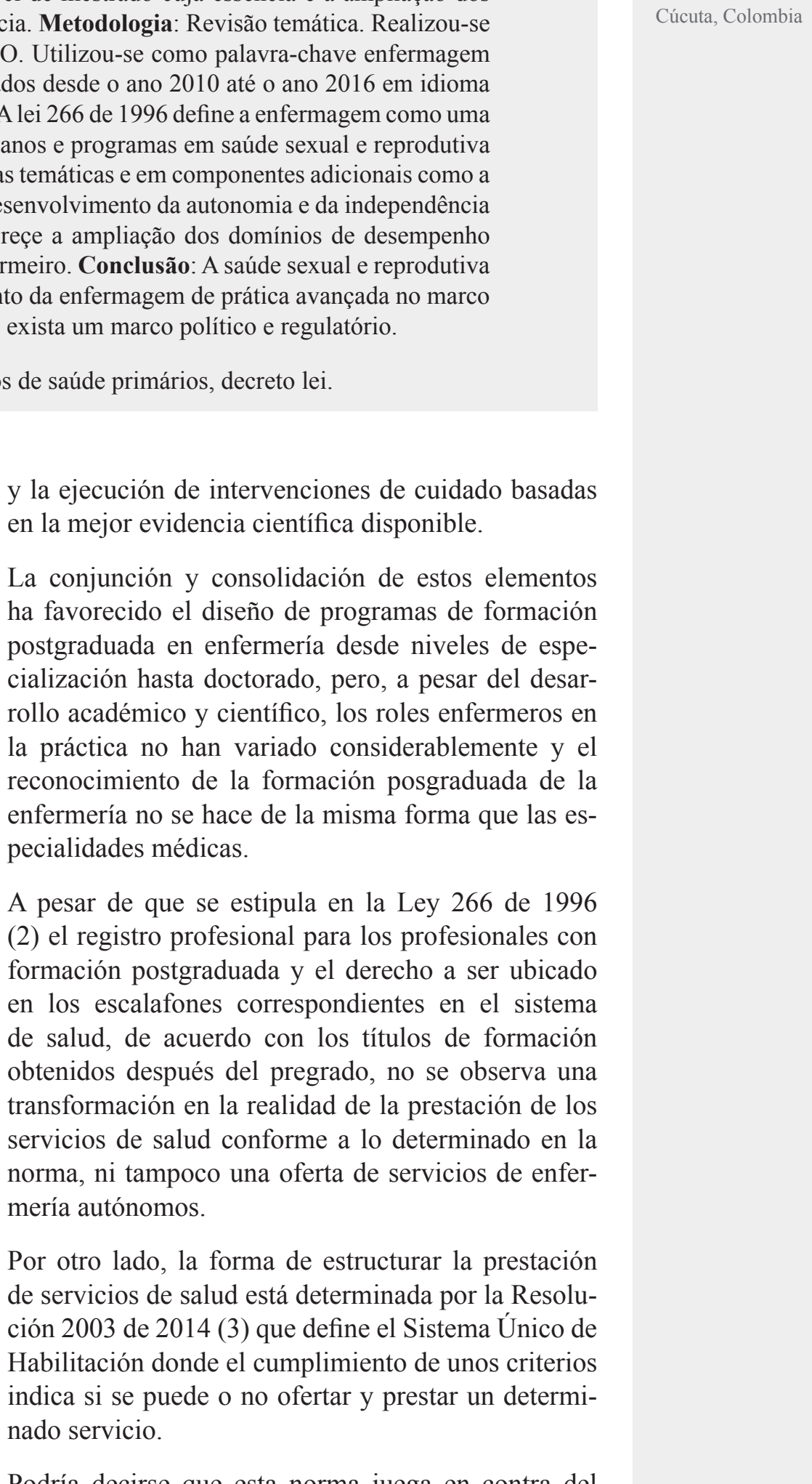

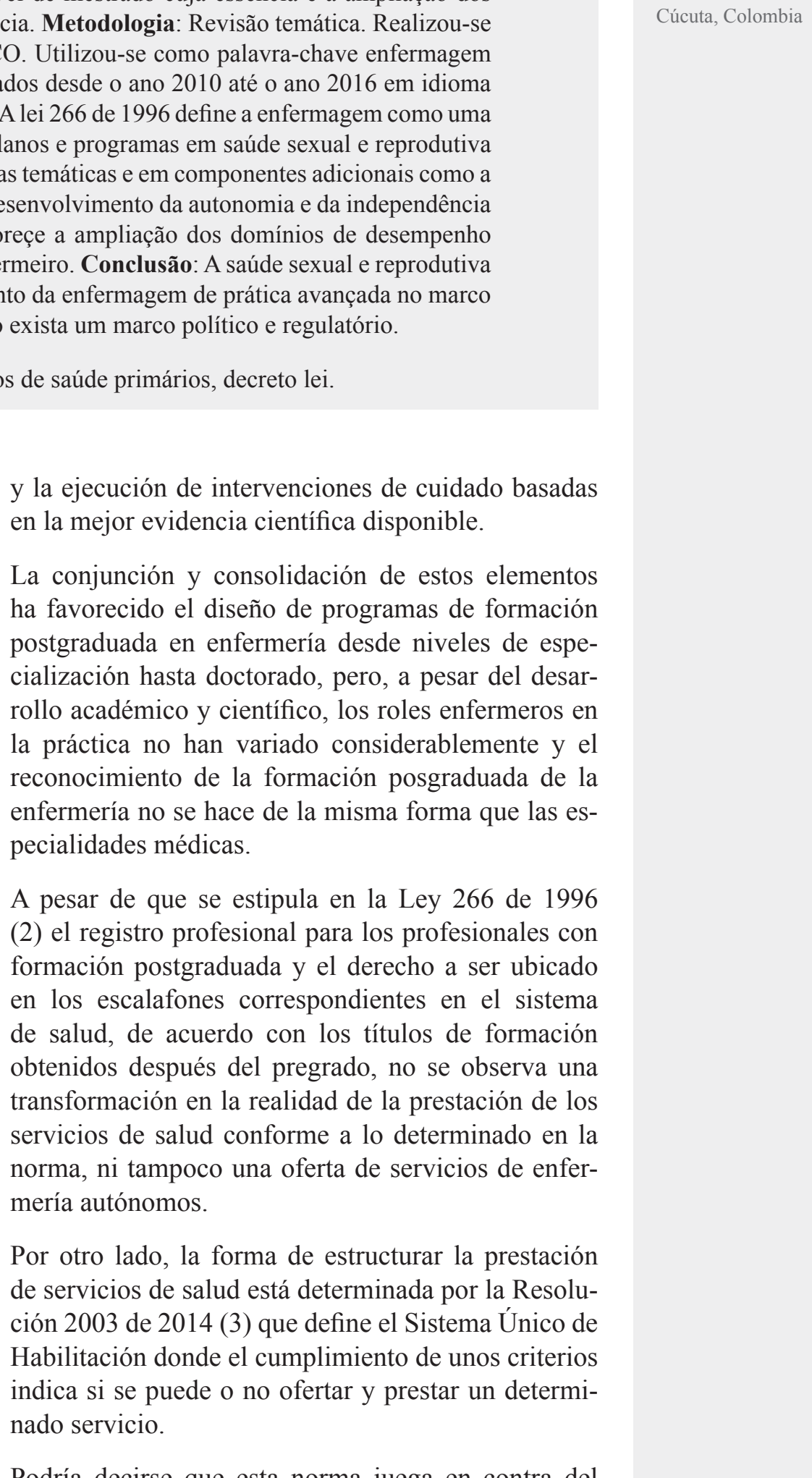

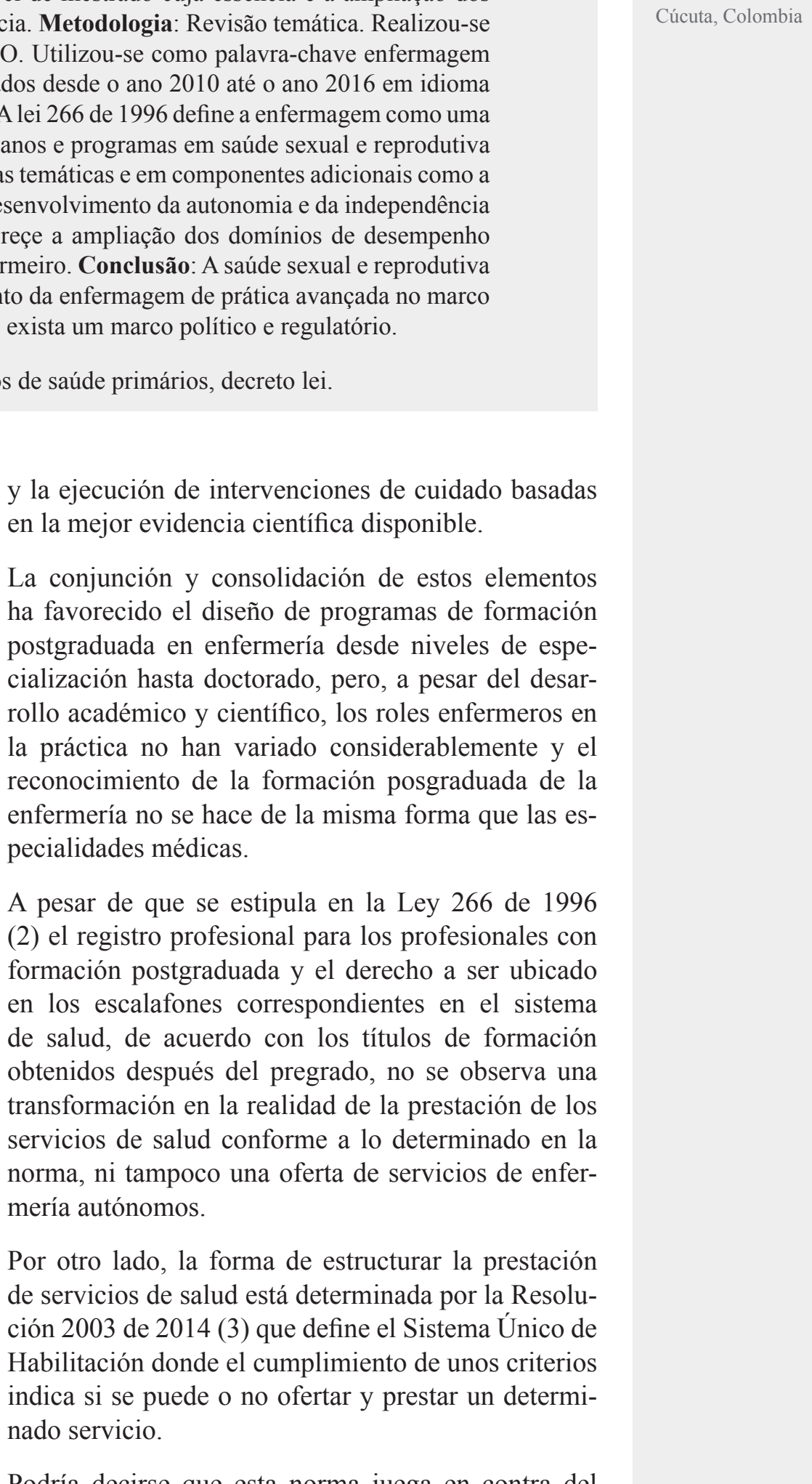

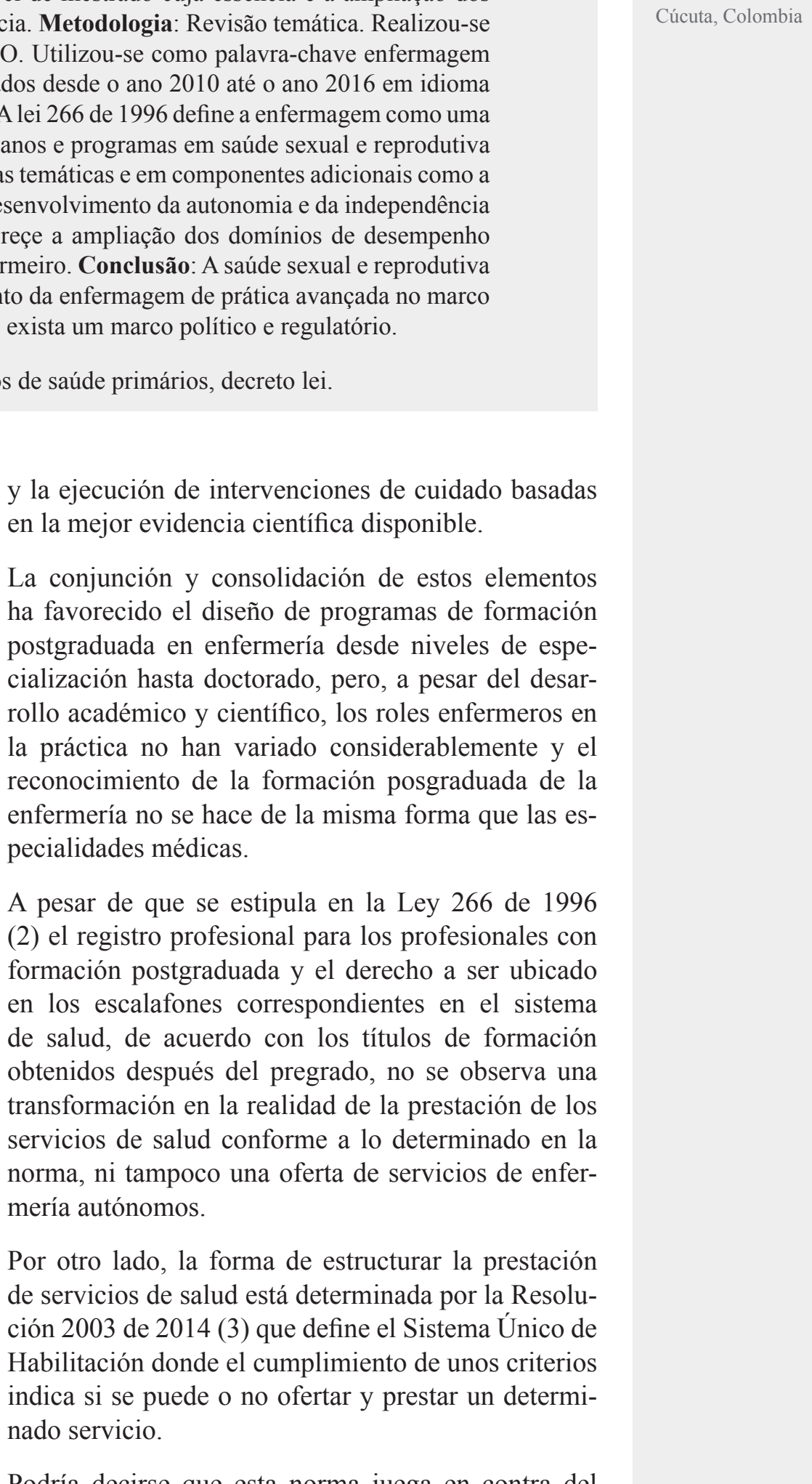

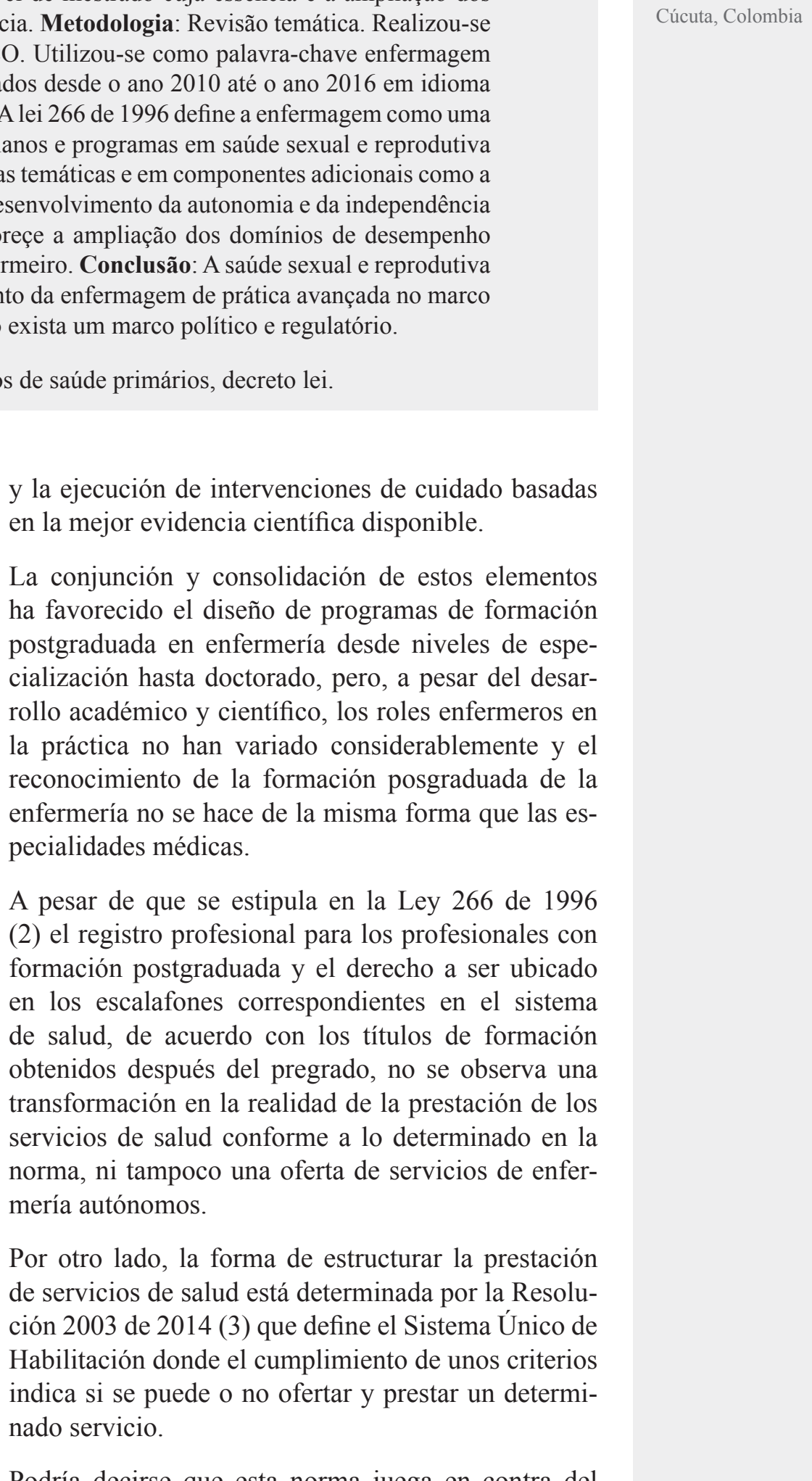

Podría decirse que esta norma juega en contra del desempeño y desarrollo académico y científico del profesional de enfermería toda vez que, para algunos 
ISSN-PRINT

1794-9831

E-ISSN 2322-7028

Vol. 14 No. 2

Jul - Dic 2017

Cúcuta, Colombia servicios, en el estándar de talento humano se prioriza la auxiliar de enfermería o se deja abierta la alternativa de que sea un profesional o un auxiliar quien esté presente, $y$, en otros, se contempla la enfermera con certificaciones, pero no se hace una exigencia de la formación especializada.

Aunque existen normas que decretan la liberalidad, autonomía e independencia de la profesión como la Ley 266 de 1996 (2) y la Ley 911 del 2004 (4), y otras como la del Sistema Único de Habilitación que limitan el quehacer enfermero, se hace necesaria la configuración de roles más avanzados de práctica en enfermería garantes de una verdadera autonomía, como sucede en otros países como Estados Unidos, Canadá, Reino Unido, España y Brasil.

La denominada enfermería de práctica avanzada (EPA) se diferencia de la práctica corriente porque implica ampliar los dominios del quehacer profesional que se derivan de la generación de nuevos roles y espacios de desempeño o por la asunción de competencias de otras profesiones como la médica (5).

Comellas (6) menciona, además, que los cambios en la pirámide poblacional -donde la población mayor de 60 años se está incrementando-, la necesidad de reajustar el abordaje de la salud y el imperativo de hacer más eficientes los sistemas de salud son motivos para que la formación y la práctica enfermera se reajuste con el desarrollo de nuevos roles que permitan satisfacer las necesidades de la población y los objetivos de los sistemas de salud.

En algunos países el desarrollo de la EPA se debe especialmente a la escasez de profesionales de la medicina (7), pero en otros surge por la necesidad de dar respuesta a las demandas de salud de la población, particularmente en escenarios extramurales (8) o en espacios diferentes a los clínicos (9).

Aunque requiere de un compromiso estatal y de un marco político y regulatorio, la EPA también requiere de nichos de desempeño. Experiencias con pacientes crónicos (10), en psiquiatría (11), neonatología y pediatría (12), maternidad (13) y cuidado intensivo (7), entre otras, indican que es necesario delimitar el campo de acción o el rol de la enfermera de práctica avanzada.

Estos roles deben ser desarrollados teniendo en cuenta competencias como el liderazgo y la gestión, el diseño y ejecución de investigación, la aplicación de los resultados a la práctica, la colaboración y el trabajo equipo -ya sea unidisciplinar, multidisciplinar o transdisciplinar-, el cuidado directo -clínico o comunitario, individual, familiar o colectivo-, el entrenamiento, la capacitación y formación continua, la consultoría y la toma de decisiones éticas (14).

La salud sexual y reproductiva (SSR) es un componente fundamental de la salud de un individuo. Se ha convertido en un hito al contemplar, para su ejercicio, la formulación de los denominados derechos sexuales y reproductivos que buscan el máximo nivel de salud posible en todos los aspectos relacionados con la sexualidad y la reproducción y garantizar de forma fundamental el derecho a la vida.

Hace parte de políticas que van desde las conferencias sobre población y desarrollo, además de objetivos de desarrollo mundial, hasta planes para mejorar las condiciones en las que se viven la sexualidad y la reproducción como la Política Nacional de Salud Sexual y Reproductiva y la de Sexualidad y Derechos Sexuales, pasando por otros planes específicos como el Plan Decenal para el Control del Cáncer en el que se incluyen los principales cánceres relacionados con estos dos aspectos.

Todos ellos, tanto las políticas como los planes, buscan, en su esencia, enaltecer la trascendencia de los aspectos sexuales y reproductivos en la salud de un individuo como generadores de satisfacción y gozo, así como de dolor, enfermedad y muerte.

Algunos indicadores de la salud sexual y reproductiva de la población muestran la complejidad que subyace en este aspecto de la salud, pues siguen sin alcanzar los resultados esperados. Ejemplo de ello es la fecundidad específica en adolescentes que, de acuerdo con la Encuesta Nacional de Demografía y Salud del año 2015 (15), bajó solamente 2.1 puntos porcentuales pasando de 19.5 en el año 2010 a 17.4 en el año 2015.

El $19 \%$ de las mujeres unidas y el $17.5 \%$ de las mujeres no unidas pero sexualmente activas, no usa métodos anticonceptivos; el $18 \%$ de las mujeres acceden a métodos de anticoncepción a través de las farmacias; las mujeres sin educación tienen una cobertura del control prenatal sólo del $69.5 \%$ y una atención del parto institucional sólo en el $63.6 \%$ de los casos (15). 
Respecto a la detección temprana de los principales cánceres relacionados con la sexualidad y la reproducción, la encuesta evidencia que apenas el $48.3 \%$ de las mujeres se ha realizado un examen clínico de la mama en el último año; entre las mujeres de 40 a 69 años, sólo el $48.1 \%$ se ha realizado una mamografía; así mismo, solamente el $34.6 \%$ de los hombres entre 50 a 69 años se ha realizado un tacto rectal y el $44.6 \%$ un antígeno prostático (15).

Adicionalmente, el $30 \%$ de los hombres y el $50 \%$ de las mujeres entre 13 a 49 años se ha realizado una prueba de VIH alguna vez en su vida. El $31.9 \%$ de las mujeres y el $22.4 \%$ de los hombres relataron haber sido víctimas de violencia física por parte de su pareja o expareja (15).

Por lo tanto, los propósitos de esta revisión son, por un lado, conceptualizar acerca de la EPA a partir de su definición, sus elementos constitutivos y las experiencias de su implementación y, por el otro, abordar las oportunidades que se identifican en el área de la salud sexual y reproductiva para el ejercicio de una enfermería de práctica avanzada en el marco de la atención primaria en salud, como contexto para el logro de la autonomía en enfermería.

\section{Metodologia}

Revisión integrativa de la literatura tipo revisión temática, para lo cual se realizó una búsqueda en las bases de datos de Lilacs, Pubmed y EBSCO. Se utilizó como palabra clave enfermería de práctica avanzada y su equivalente en inglés. Las búsquedas se limitaron a los artículos publicados desde el año 2010 hasta el año 2016, en idioma inglés, español y portugués.

Se incluyeron 26 artículos cuyo tema central fue la enfermería de práctica avanzada en aspectos como: definición del concepto, aspectos relacionados con la implementación, experiencias de implementación y desarrollo. Se excluyeron por título y resumen 53 artículos.

Posteriormente, si el artículo cumplía con el criterio de selección establecido, se procedió a descargar en texto completo para su lectura. De este segundo filtro se excluyeron 12 artículos. No se realizó evaluación de calidad de los artículos por no considerarlo pertinente con el tipo de revisión. De cada artículo incluido se extrajo la información que permitiera darle estructura a la revisión.
Adicionalmente, se realizó una búsqueda y lectura de las siguientes normas, planes, políticas, normas técnicas y guías de práctica clínica en salud sexual y reproductiva: Política Nacional de Salud Sexual y Reproductiva, Plan Decenal de Salud Pública, Plan Decenal para el Control del Cáncer, Política Nacional de Sexualidad y Derechos Sexuales y Reproductivos, Guía de Práctica Clínica para la prevención, detección temprana y tratamiento de las complicaciones del embarazo, parto y puerperio, Guía de Práctica Clínica para el manejo sindrómico de las infecciones de transmisión sexual, Modelo de Servicios Amigables para Jóvenes, Modelo de Atención a víctimas de violencia sexual, norma técnica de detección temprana de alteraciones del joven y norma técnica de planificación familiar.

Estos documentos se incluyeron para identificar elementos relacionados con la necesidad de formación o de práctica a otro nivel por parte de los profesionales de salud, en especial los enfermeros. Así mismo, se integraron elementos de las leyes de enfermería -la Ley 266 de 1993 y la Ley 911 de 2004- que mencionan aspectos relacionados con la autonomía e independencia profesional.

\section{Análisis}

\section{Soporte normativo y político para el ejercicio autónomo de la enfermería.}

La Ley 266 de 1996 (2) define la enfermería como una profesión liberal cuyos propósitos incluyen la promoción de la salud, la prevención de la enfermedad, la intervención en el tratamiento, la rehabilitación y la recuperación. Adicionalmente, esta ley plantea como competencias del profesional formular, diseñar e implementar modelos de cuidado de enfermería y como derechos proponer innovaciones a los sistemas de salud y de enfermería.

La Ley 911 de 2004 (4) establece que para que el profesional de enfermería pueda actuar con autonomía e independencia se requieren condiciones como la suficiencia de personal, infraestructura y dotación, entre otras, que, desde la perspectiva de esta revisión, sólo dan cuenta de aspectos logísticos mas no de asuntos científicos que favorezcan su crecimiento y desarrollo como disciplina.

Puede interpretarse que la disciplina debería ser autónoma en su actuar y que su formación favorecería la aplicación de intervenciones de cuidado en diferentes contextos y a diferentes grupos 
ISSN-PRINT

$1794-9831$

E-ISSN 2322-7028

Vol. 14 No. 2

Jul - Dic 2017

Cúcuta, Colombia poblacionales basadas en conocimiento propio. Sin embargo, la autonomía, entendida parcialmente como el empleo de los propios criterios para desempeñar su labor y ejercer mayor control sobre el mismo, debe ser favorecida por leyes, políticas y planes, además de los referentes teóricos propios de la disciplina, la investigación y la articulación de los resultados investigativos con la práctica (16).

Desde otro frente, el Plan Decenal de Salud Pública 2012-2021 (17) incluye, dentro de sus dimensiones de salud, la dimensión de sexualidad, derechos sexuales y reproductivos cuyo objetivo se planteó para garantizar el ejercicio pleno y autónomo de estos derechos y reducir las condiciones de vulnerabilidad mediante la atención integral.

Esta dimensión contempla dos componentes: uno de ellos es la prevención y atención integral en SSR que tiene, entre otros objetivos, implementar la atención preconcepcional, prenatal, del parto, puerperio y prevención del aborto inseguro por parte de personal calificado; promover el acceso a servicios de salud sexual y reproductiva de población adolescente, promover la atención integral a víctimas de violencia sexual y de género y prevenir y atender a las personas con riesgo o desarrollo de infecciones de transmisión sexual.

Este plan propone estrategias como el desarrollo de capacidades del talento humano en salud para la atención integral y con calidad de la mujer, antes, durante y después de un evento obstétrico y de la población objeto de esta dimensión.

Más adelante, la Política Nacional de Sexualidad, Derechos Sexuales y Derechos Reproductivos 20142021, formulada en el año 2013 (18) y que reemplazó a la antigua Política Nacional de Salud Sexual y Reproductiva, plantea como acciones la promoción de servicios de salud sexual y reproductiva con base en evidencia e investigación científicamente válida, el fortalecimiento del recurso humano en la temática sexual y reproductiva para la cualificación de la prestación de los servicios relacionados con estos aspectos de la vida de las personas y establece, como una de las principales estrategias, el fortalecimiento de la gestión dentro del sector salud y, dentro de ésta, la excelencia del talento humano y el fortalecimiento de los sistemas de investigación.

El Plan Decenal para el Control del Cáncer en
Colombia (19) formula, en sus acciones para el control del riesgo y la prevención primaria, la educación y el entrenamiento de los profesionales de la salud y la incorporación en los currículos de formación de pregrado de las profesiones de salud el desarrollo de competencias para abordar, desde los diferentes componentes de la atención en salud, a la población con riesgo de desarrollar el cáncer, a la que ya lo padece o la que está en proceso de recuperación o rehabilitación.

Como se ha visto hasta el momento, las diferentes políticas y planes reclaman, de forma tácita, la formación de profesionales de enfermería expertos para el abordaje de la sexualidad y la reproducción. Estas dimensiones son complejas y multifacéticas $\mathrm{y}$, por tanto, requieren una preparación y experticia más allá de la simple formación de pregrado y que trascienda la práctica basada en la intuición, la autoridad, la tradición o el sentido común.

El contexto político, social, cultural y epidemiológico en el que se mueve la sexualidad y la reproducción requiere de profesionales de enfermería con formación postgraduada y de una práctica avanzada que favorezca la toma de decisiones de forma autónoma para el manejo de los principales eventos relacionados con las prácticas sexuales y reproductivas a través de intervenciones de enfermería basadas en la mejor evidencia disponible.

En consecuencia, se deben generar, a su vez, preguntas de investigación que puedan ser resultas con cualquier enfoque investigativo cuyos resultados repercutan en la misma práctica y cuidado individual, familiar y colectivo. Adicionalmente, tal como lo sugiere Atehortúa (20), esta práctica autónoma debería estar basada en teorías propias que favorezcan de una forma real y tangible el ejercicio de la profesión, al igual que el uso de otras herramientas como los lenguajes estandarizados para diagnosticar, intervenir y evaluar los cuidados.

\section{Contexto general de la enfermería de práctica avanzada}

La enfermería de práctica avanzada (EPA), de acuerdo con Hernández (5), está constituida por desarrollos nuevos aplicados por profesionales de enfermería, que favorecen la ampliación de los dominios de desempeño profesional, ayudan a visibilizar el quehacer enfermero $y$ generan una verdadera autonomía profesional. 
Para Dowling (14), la EPA tiene cuatro atributos identificados en su análisis de concepto: la experiencia clínica, el liderazgo, la autonomía y el desarrollo de roles. Adicionalmente, menciona como habilidades, entre otras, el uso del conocimiento en la práctica, el juicio clínico, la habilidad para tomar decisiones y habilidades de investigación.

El liderazgo es un atributo fundamental ya que permite dar respuesta a dos asuntos disciplinares en enfermería. Uno de ellos se refiere al patrón de conocimiento socio-político, mientras que el otro tiene que ver con la gestión como área de desempeño histórico del profesional.

Jackson et al. (21) indican que es vital para enfermería emplear teorías del liderazgo que puedan ser aplicables al quehacer específico del cuidado y que permitan integrar los elementos de la disciplina que la hacen una ciencia y aquellos que la hacen un arte.

Lograr este propósito implica entender también la configuración del patrón de conocimiento sociopolítico cuya esencia es el conocimiento del contexto. Es en la medida en que se conocen los contextos -no sólo en los que se da el diario vivir de los sujetos, sino también en los que se da la práctica de la enfermeraque puede contribuirse a la transformación de las realidades de las poblaciones y de la disciplina; de ahí que se considere la formulación de políticas, planes y programas la forma de expresión de este patrón (22).

Todo lo anterior se vincula, como bien lo plantea Bautista (23), a las capacidades gerenciales que se requieren para ejercer la profesión; capacidades que no sólo se relacionan con los aspectos organizacionales sino que también involucran a los mismos sistemas de salud y el diseño de programas y proyectos para la atención en salud y el desarrollo de la enfermería.

Dentro de los antecedentes de la EPA se indica que los altos niveles de formación y la experiencia clínica determinan la aparición de esta forma de hacer enfermería. Respecto al nivel de formación a partir del cual se podría considerar la posibilidad de ejercer la enfermería de práctica avanzada, son varios los autores $(14,24-27)$ que consideran que es el nivel de maestría el adecuado para comenzar. Adicionalmente, Fitzgerald (28) plantea que la demanda de enfermeras preparadas para atención avanzada aumenta en la medida en que aumenta la formación de las mismas.
La enfermería de práctica avanzada podría tener un nicho especial en Colombia con la formulación de la Ley 1438 de 2011 (29) que propone la Atención Primaria en Salud (APS) como el eje central de la atención en el sistema actual donde la promoción y la prevención, a lo largo de los diferentes ciclos vitales, se convierten en dos pilares fundamentales de actuación de la enfermería de práctica avanzada.

Así lo confirma la Política de Atención Integral en Salud (30) donde una de las cuatro estrategias centrales es la APS, ya que se considera una estrategia integradora entre las necesidades de la población, la respuesta del sistema y los recursos disponibles para generar bienestar y abordar el riesgo en salud de los individuos y las comunidades.

También lo plantea Atehortúa (20) cuando considera que los modelos de atención primaria en salud evidencian mejores resultados en salud, disminución de costos, mayor eficiencia y mayor satisfacción.

En consecuencia, se podría contribuir y dar respuesta a la recomendación de la Organización Mundial de la Salud (28) de incrementar el número de enfermeras de práctica avanzada para apoyar los sistemas de salud basados en la atención primaria y que favorezcan el objetivo de la cobertura universal en salud.

Para abrirse a la posibilidad de implementar y llevar a la realidad contextual una enfermería de práctica avanzada se requieren cambios sociales en la forma de abordar el cuidado de la salud a los individuos y colectivos desde sus lugares de residencia y de diario vivir hasta los espacios institucionales.

Cambios políticos que incluyan el reconocimiento diferencial de la enfermería como disciplina capaz de gestionar el cuidado integral de las personas y las comunidades y la participación activa de los grupos colegiados y de los mismos enfermeros en la construcción de las políticas de salud y de cuidado.

Igualmente, cambios educativos para garantizar una formación basada en la evidencia, con el uso de teorías de mediano rango para ser llevadas a la práctica, un uso de lenguaje estandarizado, un fuerte componente de investigación que favorezca tanto la comprensión de las diferentes realidades como la explicación, la predicción y la efectividad de las intervenciones de cuidado, además de cambios profesionales que 
ISSN-PRINT

1794-9831

E-ISSN 2322-7028

Vol. 14 No. 2

Jul - Dic 2017

Cúcuta, Colombia mejoren las relaciones entre colegas y que favorezcan el crecimiento conjunto de la profesión.

Debe existir también un amparo legal que propicie las acciones enfermeras desde la promoción, pasando por el diagnóstico y llegando hasta la rehabilitación. Esto implicaría, entonces, el desarrollo de competencias políticas que contribuyan a la participación en escenarios donde se toman decisiones de regulación en cuestiones de salud y de recursos humanos para la prestación de servicios de salud. Adicionalmente, la participación en organizaciones gremiales que defienden y visibilizan los aportes de la disciplina a la sociedad (31).

Existen alrededor del mundo varias experiencias en el desarrollo de la EPA. Estados Unidos, por ejemplo, tiene cuatro roles oficialmente identificados: la enfermera practicante, la especialista en enfermería clínica, la enfermera anestesista y la enfermera partera. También se cuenta con seis poblaciones y/o áreas identificadas: el individuo y la familia, el adulto y la gerontología, los neonatos, la pediatría, la salud de la mujer y de género y la salud mental y psiquiatría (14).

En los países escandinavos, por su parte, se ha considerado la EPA para el cuidado de los adultos mayores (32). Morilla et al. (33) realizaron una revisión sistemática en la que incluyeron quince estudios y concluyeron que, en escenarios de cuidado a largo plazo, la EPA ha dado buenos resultados reduciendo las readmisiones e incrementando la satisfacción de los pacientes y los cuidadores, pero advierte que, dada la participación de la enfermera en un equipo multidisciplinario, es difícil discernir si el efecto se atribuye sólo a su práctica.

En Brasil, Goncalves (34) reportó una experiencia de EPA en un servicio de oncología con la incorporación del enfermero especialista en oncología pediátrica y cuyas funciones están basadas en cuatro pilares esenciales: la asistencia cualificada, la educación y entrenamiento al paciente, la familia y otros profesionales; la investigación y la gestión.

En Indonesia, la enfermería psiquiátrica es vista como EPA pero, de acuerdo a Wardani (11), requiere de unos ajustes en el proceso de formación que garanticen su desempeño autónomo.

Coyne et al. (35) evidenciaron en su estudio que los escenarios donde existía una figura de enfermera de práctica avanzada diferían de aquellos donde no existía, especialmente en desenlaces como tasas de readmisión de pacientes, tiempos de espera, continuidad del cuidado y carga del cuidado.

Concluye que, para satisfacer las demandas en salud que continuamente están cambiando, promover la atención centrada en la persona y mejorar la prestación de los servicios de salud y de enfermería para que sean concebidos como de calidad, deben desarrollarse y apoyarse más roles especializados y avanzados en la formación y práctica enfermera en el ámbito asistencial clínico y comunitario.

Otro aspecto fundamental en la EPAes la transformación de los programas de formación enfermera. Se requiere, como propone Zanetti (25), adoptar estrategias de formación que incluyan la práctica avanzada, con modelos teóricos propios de enfermería, una fuerte fundamentación de conocimiento basado enlaevidencia y la capacidad de dirigir equipos interdisciplinarios para favorecer la toma de decisiones creativas, eficaces y de bajo costo para la población.

De Bortolli (26) indica que el desarrollo de las competencias de la EPA y las oportunidades de formación permanente deben ser asumidos y gestionados por las instituciones de educación superior.

Así entonces, hay quienes indican que no puede confundirse la práctica de una enfermera especialista o magister con lo que debería ser una enfermera de práctica avanzada.

La formación posgraduada en enfermería en este país aún no tiene el reconocimiento social y político requerido para ejercer con completa autonomía e independencia la aplicación del proceso de atención en enfermería con énfasis en la prescripción de intervenciones de cuidado efectivas, pues aún es notoria la hegemonía médica en la prestación de los servicios de salud y los planes de cuidado de enfermería todavía siguen siendo, para muchas instituciones de salud y muchos profesionales enfermeros, las órdenes médicas, fenómeno que perpetua la imagen subyugada de este profesional y su rol como auxiliar o apoyo del personal médico.

Como lo indica East (36), las enfermeras pueden basar su práctica en la evidencia científica y sus acciones pueden ser interpretadas como de práctica avanzada, pero no cumplen con todos los determinantes 
ampliamente documentados en la literatura como la autonomía, la expansión de dominio de desempeño, la educación y la investigación.

La EPA puede también ser percibida como una oportunidad para las enfermeras ya que, como demuestra Shearer (37), el resultado es un cambio positivo derivado del mejoramiento de las habilidades de evaluación clínica, el incremento en la confianza, la importancia del trabajo en red y el incremento de la autonomía.

\section{Articulación de la salud sexual y reproductiva y la enfermería de práctica avanzada}

En Colombia existen herramientas que favorecen el desempeño y la formación de la enfermería de práctica avanzada en la salud sexual y reproductiva.

La Política de Sexualidad define seis temas en los que se puede tener un desempeño autónomo: la planificación familiar y la anticoncepción, la salud sexual y reproductiva de jóvenes y adolescentes, el manejo de las infecciones de transmisión sexual, la prevención y detección temprana del cáncer de cérvix, la prevención, diagnóstico temprano, manejo y seguimiento a víctimas de violencia sexual y la salud materna.

Pero no solamente estos podrían ser los eventos a abordar por parte de una enfermera de práctica avanzada. Otros que se incluirían son: la prevención y detección temprana del cáncer de mama, la prevención y detección temprana del cáncer de próstata, el abordaje de las diversas orientaciones sexuales para garantizar prácticas de autocuidado seguras y responsables, el cuidado de la mujer en trabajo de parto, la atención del parto y el postparto y el seguimiento y acompañamiento domiciliario o institucional para la transición de la mujer hacia la maternidad.

Todos estos eventos tienen la posibilidad de ser abordados a través de la estrategia de la Atención Primaria en Salud como puerta de entrada al sistema y a servicios de mayor complejidad, entendiendo el primer nivel de atención como alternativa de acceso a los servicios de salud para la mayoría de la población, aunque los demás niveles de complejidad también se configuran como escenarios clínicos potenciales para el ejercicio de una enfermería de práctica avanzada.

Dentro de la planificación familiar y la anticoncepción, la consulta pre-concepcional es una actividad para la que se conocen intervenciones prioritarias que son costo-efectivas y que pueden ser ejecutadas por enfermeras de práctica avanzada (38).

Adicionalmente, se cuenta con el componente preconcepcional desarrollado por la estrategia de Atención Integral a las Enfermedades Prevalentes de la Infancia (39) que concibe el cuidado preconcepcional como un componente crítico en la atención de las mujeres en edad reproductiva y cuya atención puede optimizar los resultados perinatales y maternos.

La Resolución No. 0769 de 2008 (40), aunque ya faculta a las enfermeras para que hagan consultas de anticoncepción de ingreso y los respectivos controles, autoriza la inserción del DIU y del implante subdérmico y permite la provisión de anticonceptivos a través de fórmula, no provee todo el marco de acción e intervenciones que podría ejecutar una enfermera en un contexto de práctica avanzada. Intervenciones como la inserción y retiro del endoceptivo hormonal podrían ser parte del resorte de la enfermera, con lo que se lograría un completo dominio de la anticoncepción y podría pasar a ser un campo de acción exclusivo de esta disciplina.

Con respecto a la salud sexual y reproductiva de los jóvenes y adolescentes, actualmente hay dos estrategias que posibilitan que la enfermera sea de práctica avanzada. Una de ellos la constituyen los servicios amigables para jóvenes y adolescentes; el otro es la norma técnica de detección de alteraciones del joven.

En el primero se establece que los profesionales que prestan servicios a jóvenes y adolescentes deben poseer ciertas aptitudes que les permita evaluar, tomar decisiones y realizar intervenciones (41).

En el segundo, a pesar de que se indica que la consulta sea realizada por médico, es posible que ésta pueda ser ejecutada por una enfermera de práctica avanzada toda vez que las actividades de la consulta hacen parte del quehacer enfermero, pues este profesional estaría en capacidad de valorar, más allá de los aspectos físicos, aquellos componentes psicológicos, sociales y culturales que determinan las prácticas y hábitos de este grupo poblacional (42).

Además, podría tener la potestad de ordenar los exámenes estipulados en la norma para laidentificación
E-ISSN 2322-7028

Vol. 14 No. 2

Jul - Dic 2017

Cúcuta, Colombia 
ISSN-PRINT

1794-9831

E-ISSN 2322-7028

Vol. 14 No. 2

Jul - Dic 2017

Cúcuta, Colombia temprana de riesgos y, adicionalmente, realizar las remisiones pertinentes a otros profesionales para garantizar el manejo integral del joven sin perder la articulación y seguimiento del programa y de los adolescentes y jóvenes que acceden a él.

El enfoque de riesgo, como estrategia de análisis poblacional, puede ser aplicado a la consulta de los jóvenes y puede ser ejecutado por enfermería.

En relación con las infecciones de transmisión sexual, este tópico es transversal a todas las temáticas, puesto que cubre desde la consulta pre-concepcional hasta la atención a víctimas de violencia sexual, en cuyo caso se justifica mucho más la incorporación de este evento en el quehacer de la enfermera en un escenario de práctica avanzada.

Así mismo, la elaboración de la Guía de Práctica Clínica para el abordaje sindrómico de los pacientes con estas infecciones (43) facilita aún más la oportunidad de que sean las enfermeras las responsables de orientar las intervenciones sobre este evento.

No es solamente la asesoría pre y post prueba para el VIH que ya se viene haciendo en muchas instituciones de salud, ni tampoco el manejo de las lesiones condilomatosas: es incluir en el quehacer enfermero el abordaje integral de todas las infecciones, asunto que implicaría abrir un capítulo en Colombia alrededor de la prescripción farmacológica por parte de enfermería, probablemente con algunas restricciones, pero seguramente podría configurarse realmente un programa de atención integral a personas que adquieren una infección y que se convierten en un riesgo potencial para continuar con la cadena de transmisión.

En la prevención y detección temprana del cáncer de cérvix son las enfermeras de práctica avanzada las llamadas a evidenciar su capacidad de mejorar los indicadores relacionados con coberturas, acceso a diagnóstico y el inicio temprano del tratamiento.

Históricamente ha sido enfermería la disciplina más representativa en lo que a manejo de los programas de tamización para cáncer de cérvix se refiere. Sin embargo, en los últimos años la figura de la histocito-tecnóloga ha venido a ocupar espacios que inicialmente pertenecían a enfermería. Y aunque las normas de habilitación siguen contemplando a las enfermeras junto con los médicos y los bacteriólogos como los únicos profesionales autorizados para la toma de citologías cérvico-uterinas, es indispensable recuperar terrenos de actuación.

En un contexto de práctica avanzada, el profesional de enfermería debe ser capaz de interpretar los resultados de una citología y definir la conducta más adecuada de seguimiento, incluyendo la remisión a los diferentes procedimientos.

Pero se podría ir más allá e incluir la realización de la colposcopia y la toma de la biopsia, como ocurre en otros países. De igual forma, la enfermera podría realizar la tamización denominada de inspección visual en un escenario de atención primaria para mejorar la cobertura y el acceso al tratamiento de lesiones precancerosas, dado que ya se cuenta con una guía de práctica clínica (44) para el manejo de estas lesiones y que incluye, como recomendación fuerte a favor, la tamización con pruebas de inspección visual con ácido acético y lugol.

En cuestiones de salud materna, la Guía de práctica clínica para la prevención, detección temprana y tratamiento de las complicaciones del embarazo, parto o puerperio (45) establece como recomendación grado A que el control prenatal puede ser realizado por médico o enfermera capacitada o especializada.

Así mismo, existen varios instrumentos (46 - 49) desarrollados en torno a la transición de convertirse en madre que pueden favorecer el quehacer autónomo de la enfermera en los programas de control prenatal, parto y postparto -más allá de los aspectos biológicos- y evidenciar óptimos resultados, como lo sugiere Johantgen et al. (50) en su revisión sistemática acerca de los efectos de la enfermera de práctica avanzada comparada con los profesionales médicos, en desenlaces como cuidado del trabajo de parto, uso de analgesia epidural, realización de episiotomía, resultado de Apgar del recién nacido, peso al nacer y admisiones al servicio de cuidados intensivos neonatales.

Indican estos autores (50) que los resultados relacionados con la atención del parto fueron más bajos para los médicos, no hubo diferencias en los desenlaces relacionados con el recién nacido, pero fueron más altos los resultados para las enfermeras en la lactancia materna y tuvieron más pocas laceraciones perineales.

En lo que tiene que ver con violencia sexual, este es un asunto transversal a todas las posibles atenciones e 
intervenciones de la enfermera de práctica avanzada en la salud sexual y reproductiva. De acuerdo con el Modelo de Atención a Víctimas de Violencia Sexual (51), un profesional entrenado debe sospechar la violencia sexual a través de ciertos signos y síntomas relacionados en cualquier escenario de atención.

Un profesional de enfermería de práctica avanzada puede ejecutar muchas de las actividades contempladas en la atención a una víctima de violencia sexual y obtener resultados adecuados, tal como lo demuestra Dos Reis (52), donde los enfermeros que atendieron las víctimas evidenciaron coherencia entre los diagnósticos de enfermería, las intervenciones y los protocolos del programa.

Respecto a la prevención y detección temprana del cáncer de mama, en un escenario de práctica avanzada de la enfermería, se deben ejecutar acciones que impliquen la realización del examen de la mama de forma anual y la remisión a la realización de la mamografía, así como el seguimiento y la derivación al manejo pertinente de acuerdo con el resultado de la misma.

Para la prevención y detección temprana del cáncer de próstata, la realización del tacto rectal y el ordenamiento del antígeno prostático podrían contemplarse en el actuar de este rol de la práctica enfermera, además de articularse y ejecutarse en el contexto de la atención primaria en salud, toda vez que se desconoce la cobertura de la prueba de tamización al no existir un programa estructurado para esta patología que se ha convertido en una causa importante de muerte por cáncer para los hombres (53).

Es claro que el panorama nacional favorece una enfermería de práctica avanzada, pero la existencia de las actuales normas y planes no son suficientes para lograr este gran paso hacia una enfermería autónoma, liberal y con expansión de sus dominios profesionales. Aunque es una tendencia mundial, se requieren políticas, regulaciones, voluntad estatal y movilización profesional y gremial que soporten su aparición, desarrollo, mantenimiento y resultados.

Como indica Schober (54), la enfermería de práctica avanzada no puede desarrollar su rol basada solamente en asuntos teóricos y académicos porque esto no garantiza su rol en la práctica. Es por esto que se deben generar unos lineamientos estatales organizados y planeados que favorezcan un marco conceptual y político para la puesta en marcha de esta forma de brindar los cuidados.

Aunque para autores como Ryley (55) esta forma de la enfermería no debe ser vista como un rol sino como un nivel de práctica que debe ser encabezado por el Estado ya que está demostrado el impacto que tiene en la entrega de cuidados de alta calidad.

\section{Conclusiones}

La disciplina de enfermería, en Colombia, posee un marco jurídico que la define como autónoma e independiente, criterios que deben ser validados en la práctica a través de un quehacer basado en cuatro elementos fundamentales: la enfermería basada en la evidencia, el uso de teorías propias de la disciplina, el uso de un lenguaje estandarizado y la generación de nuevo conocimiento a través de la investigación.

La salud sexual y reproductiva, como un componente primordial y fundamental de la salud de un individuo y de una población, requiere abordajes y comprensiones más profundas, científicas y fundamentadas para lograr entender los complejos matices que se dan en las prácticas sexuales y reproductivas que conllevan a la persistencia de riesgos, tal como se observa en la situación de salud del país.

La enfermería de práctica avanzada como tendencia mundial es una oportunidad para el desarrollo de nuevos roles para el quehacer de los profesionales de enfermería de Colombia y como propuesta de la OMS en el marco de la atención primaria en salud para cumplir con el mandato de acceso y atención a los servicios de salud para todos y mejorar las coberturas de los programas relacionados con la sexualidad y la reproducción.

La expansión del dominio de práctica es uno de los principales elementos que caracteriza la enfermería de práctica avanzada y el área de la salud sexual y reproductiva es el terreno ideal para sembrar, cosechar y recolectar los frutos de una enfermería que promueve la salud, previene la enfermedad, detecta precozmente, diagnóstica, prescribe acciones de cuidado, realiza seguimiento y participa en la rehabilitación, en articulación con otros profesionales pero siendo la puerta de entrada a un sistema de salud que garantiza un acceso real a los servicios y al cuidado para todos. 
ISSN-PRINT

$1794-9831$

E-ISSN 2322-7028

Vol. 14 No. 2

Jul - Dic 2017

Cúcuta, Colombia

\section{Conflicto de intereses}

El autor declara no tener ningún conflicto de intereses.

\section{Referencias bibliográficas}

1. Camargo IL, Caro CV. El papel autónomo de enfermería en las consultas. av. enferm. 2010; 28(1): 143-150.

2. República de Colombia. Congreso de Colombia. Ley 266 de 1996, enero 25, por la cual se reglamenta la profesión de enfermería en Colombia y se dictan otras disposiciones. Diario oficial $\mathrm{N}^{\circ} 42.710$. Bogotá: El Ministerio; 1996.

3. República de Colombia. Ministerio de Salud y Protección Social. Resolución 2003 de 2014, mayo 28, por la cual se definen los procedimientos y condiciones de inscripción de los Prestadores de Servicios de Salud y de habilitación de servicios de salud. Bogotá: El Ministerio; 2014.

4. República de Colombia. Congreso de Colombia. Ley 911 de 2004, octubre 5, por la cual se dictan disposiciones en materia de responsabilidad deontológica para el ejercicio de la profesión de Enfermería en Colombia, se establece el régimen disciplinario correspondiente y se dictan otras disposiciones. Diario oficial No 45.693. Bogotá: El Ministerio; 2004.

5. Hernández JF. ¿Qué significa enfermería de práctica avanzada hoy y aquí? RIdEC [Internet]. 2011 [Consultado 29 abr 2016]; 4(2): 31-33. Disponible en: http://enfermeriacomunitaria.org/web/attachments/article/237/RIdEC.v4n2.31.Especialidades-3.pdf

6. Comellas-Oliva M. Construcción de la enfermera de práctica avanzada en Catalunya (España). REBEn [Internet]. 2016 [Consultado 28 dic 2016]; 69(5): 991-995. Disponible en: http://dx.doi. org/10.1590/0034-7167.2016690507

7. Jackson A, Carberry M. The advance nurse practitioner in critical care: a workload evaluation. Nurs Crit Care [Internet]. 2014 [Consultado 28 dic 2016]; 20(2): 71-77. Disponible en: doi: 10.1111/nicc.12133

8. Sanchez-Martin C. Cronicidad y complejidad: nuevos roles en Enfermería. Enfermeras de Práctica Avanzada y paciente crónico. Enferm. Clin. 2014; 24(1): 79-89.

9. Kirk H. The role of advanced nursing practice in occupational health. Occup Med [Internet]. 2012 [consultado 28 dic 2016]; 62: 574-577. Disponible en: doi:10.1093/occmed/kqs144

10. Appleby $\mathrm{C}$, Camacho R. Retos y oportunidades: aportaciones de la enfermera de práctica avanzada en la cronicidad. Aprendiendo de las experiencias. Enferm Clin. 2014; 24(1): 90-98.

11. Wardani Y. The competencies, roles and scope of practice fo advanced psychiatric nursing in Indonesia. Nurse Media Journal of Nursing [Internet]. 2013 [ consultado 13 may 2016]; 3(2): 631-648. Disponible en: doi: 10.14710/nmjn.v3i2.6004

12. Morgan $C$, Barry C, Barnes K. Master's programs in advanced nursing practice: new strategies to enhance course design for subspecialty training in neonatology and pediatrics. Adv Med Educ Pract. [Internet]. 2012 [consultado 13 may 2016]; (3): 129-137. Disponible en: https://www.ncbi.nlm.nih. gov/pmc/articles/PMC3650880/

13. Dole D, Nypaver C. Nurse-Midwifery: Art and Science. Nurs Clin N Am [Internet]. 2012 [28 dic 2016]; 47:205-213. Disponible en: doi:10.1016/j.cnur.2012.02.008

14. Dowling M, Beauchesne M, Farrelly F, Murphy K. Advanced practice nursing: a concept analysis. Int J Nurs Pract.[Internet]. 2013 [consultado 18 may 2016]; 19 (2): 131-140. Disponible en: doi:10.1111/ ijn. 12050.

15. República de Colombia. Profamilia. Ministerio de Salud y Protección Social. Resumen ejecutivo. Encuesta Nacional de Demografía y Salud. Colombia. 2015. Bogotá: Profamilia. 96 p.

16. Rodríguez N. Manual de sociología de las profesiones. Textos docentes 343. [Internet]. Barcelona: Universidad de Barcelona; 2008 [ consultado 16 may 2016]; 148 p. Disponible en: http://www.publicacions.ub.edu/refs/indices/07061.pdf 
17. República de Colombia. Ministerio de Salud y Protección Social. Plan Decenal de Salud Pública 20122021. Mayo 28 del 2013. Bogotá: El Ministerio. 456 p.

18. República de Colombia. Ministerio de Salud y Protección Social. Profamilia. Fondo de Población de Naciones Unidas. Organización Internacional para las Migraciones. Política Nacional de Sexualidad, Derechos Sexuales y Derechos Reproductivos. 2014.Bogotá: El Ministerio. 148 p.

19. República de Colombia. Ministerio de Salud y Protección Social. Instituto Nacional de Cancerología. Plan Decenal para el Control del Cáncer en Colombia, 2012-2021. 2012. Bogotá: El Ministerio. 124 p.

20. Atehortúa GM, Soto M, López ME, Buitrago LA. La práctica avanzada en enfermería: una oportunidad para la promocion de la salud y la prevención de la enfermedad en Colombia. Revista Cultura del Cuidado 2014; 11(1): 60-69.

21. Jackson JP, Clements PT, Averill JB, Zimbro K. Patterns of knowing: proposing a theory for nursing leadership. Nurs Econ. 2009; 27(3): 149-159.

22. Osorio JH. Patrón de conocimiento socio-político en enfermería: reflexiones conceptuales. Rev Cuid. 2016; 7(2): 1352-7.

23. Bautista GO. Tendencias y retos de enfermería en la gerencia de los servicios de salud en el ámbito mundial, nacional y regional. Rev. cienc. cuidad. 2014; 11(1): 68-86.

24. Galao R. Enfermería de Práctica Avanzada en España: ahora es el momento. Index Enferm 2009; 18(4): 221-223.

25. Zanetti ML. La práctica de enfermería avanzada: estrategias para la formación y creación de conocimiento. Rev. Latino-Am. Enfermagem [Internet]. 2015 [consultado 18 may 2016]; 23(5): 779-780. Disponible en: DOI: 10.1590/0104-1169.0000.2614

26. De Bortoli SH, Zug KE. Fomentando el papel de la práctica avanzada de enfermería en América Latina. Rev. Bras. Enferm. [Internet]. 2014 [consultado 13 may 2016]; 67(5): 677-678. Disponible en: http://dx.doi.org/10.1590/0034-7167.2014670501

27. Silvan GC, Lima F, Ferreira MA, Alvarez AM. Professional Master's Degree: potential contribution to advanced practice nursing. Rev Bras Enferm [Internet]. 2015 [consultado 13 may 2016]; 68(6): 874877. Disponible en: http://dx.doi.org/10.1590/0034-7167.2015680626i

28. Fitzgerald C, Kantrowitz I, Katz J, Hirsch A. Advanced practice nursing education: challenges and strategies. Nursing Research and Practice [Internet]. 2012 [consultado 13 may 2016]; Article ID 854918; 1-8. Disponible en: doi:10.1155/2012/854918

29. República de Colombia. Congreso de la República. Ley 1438 de 2011. enero 19, por medio de la cual se reforma el Sistema General de Seguridad Social en Salud y se dictan otras disposiciones. Bogotá: El Congreso; 2011.

30. República de Colombia. Ministerio de Salud y Protección Social. Política de Atención Integral en Salud. 2016. Bogotá: El Ministerio. 97 p.

31. Kostas EA, Thanavaro J, Arvidson C, Morrit LF. Advanced practice nursing: shaping health through policy. J Am Assoc Nurse Pract [Internet]. 2015 [consultado 13 may 2016]; 27(11): 11-20. Disponible en: doi: 10.1002/2327-6924.12192

32. Põlluste K, Routasalo P, Fagerström L, Wagner L. Advanced nursing practice for older people. Nurs Res PracT. [Internet]. 2013 [consultado 13 may 2016]; 2013: 860167. Disponible en: https://www. ncbi.nlm.nih.gov/pmc/articles/PMC3745979/

33. Morilla JC, García S, Martín FJ, Kaknani S, Campos AL, Caro J, et al. A systematic review of the effectiveness and roles of advanced practice nursing in older people. International Journal of Nursing Studies [Internet]. 2016 [consultado 31 may 2016]; 53: 290-307. Disponible en: http://dx.doi. org/10.1016/j.ijnurstu.2015.10.010

34. Gonçalves C, Duarte AM, Santos AS, Bonfietti D, Porto D, dos Santos J, et al. Clinical nurse specialist: a model of advanced nursing practice in pediatric oncology in Brazil. Rev. esc. enferm. USP [Internet]. 2013 [consultado 13 may 2016]; 47(6): 1426-1430. Disponible en: DOI: 10.1590/S0080623420130000600025

35. Coyne I, Comiskey CM, Lalor JG, Higgins A, Elliott N, Begley C. An exploration of clinical practice 
ISSN-PRINT

$1794-9831$

E-ISSN 2322-7028

Vol. 14 No. 2

Jul - Dic 2017

Cúcuta, Colombia

in sites with and without clinical nurse or midwife specialists or advanced nurse practitioners, in Ireland. BMC Health Serv Res. [Internet]. 2016 [consultado 02 jun 2016]; 16:151-168. Disponible en: DOI 10.1186/s12913-016-1412-8

36. East LA, Arudo J, Loefler M, Evans CM. Exploring the potential for advanced nursing practice role development in Kenya: a qualitative study. BMC Nursing [Internet]. 2014 [consultado 13 may 2016]; 13(33): 2-11. Disponible en: http://www.biomedcentral.com/1472-6955/13/33

37. Shearer D, Adams J. Evaluating an advanced nursing practice course: student perceptions. Nursing Standard [Internet]. 2012 [consultado 13 may 2016]; 26(21): 35-41. Disponible en: http://dx.doi. org/10.7748/ns2012.01.26.21.35.c8888

38. Coffey K, Shorten A. The challenge of preconception counseling: using reproductive life planning in primary care. Journal of the American Association of Nurse Practitioners [Internet]. 2014 [consultado 31 may 2016]; 26(5): 255-262. Disponible en: doi: 10.1002/2327-6924.12054

39. República de Colombia. Ministerio de Salud y Protección Social Social. Sociedad Colombiana de Pediatría. Organización Panamericana de la Salud. Curso clínico. Atención integrada a las enfermedades prevalentes de la infancia. 2012. Bogotá: El Ministerio. 819 p.

40. República de Colombia. Ministerio de la Protección Social. Resolución 0769 de 2008. Marzo 03, por medio de la cual se adopta la actualización de la Norma Técnica para la Atención en Planificación Familiar a Hombres y Mujeres establecida en la Resolución 412. Bogotá: El Ministerio, 2008.

41. República de Colombia. Ministerio de Salud y Protección Social. Fondo de Población de las Naciones Unidas. Servicios de salud amigables para jóvenes y adolescentes 2008. Bogotá: UNFPA, 2008.

42. República de Colombia. Ministerio de Salud. Norma técnica para la detección temprana de las alteraciones del desarrollo del joven de 10 a 29 años. Bogotá: El Ministerio, 2000.

43. Gaitán HG, Rodríguez AE, Arévalo I, Ángel E, López HE, Estrada JS, et al. Guía de práctica clínica para el manejo sindrómico de los pacientes con infecciones de transmisión sexual y otras infecciones del tracto genital-2013. Rev Colomb Obstet Ginecol 2013; 64(2): 126-177.

44. República de Colombia. Ministerio de Salud y Protección Social. Colciencias. Instituto Nacional de Cancerología. Guía de práctica clínica para la detección y manejo de lesiones precancerosas de cuello uterino. Bogotá: El Ministerio, 2014.

45. Amaya J, Díaz LA, Cardona A, Osorio D, Barrera A, Rubio JA, et al. Guía de práctica clínica para la prevención y detección temprana de las alteraciones del embarazo. Rev Colomb Obstet Ginecol. 2013; 64(3): 245-288.

46. Matthey S. Assessing the experience of motherhood: The Being a Mother Scale (BaM-13). J Affect Disord. [Internet]. 2011 [consultado 31 may 2016]; 128: 142-152. Disponible en: doi:10.1016/j. jad.2010.06.032

47. Bienfait M, Maury M, Haquet A, Faillie JL, Franc N, Combes C, et al. Pertinence of the self-report mother-to-infant bonding scale in the neonatal unit of a maternity ward. Early Hum Dev. [Internet]. 2011 [consultado 31 may 2016]; 87(4): 281-7. Disponible en: doi:10.1016/j.earlhumdev.2011.01.031

48. Hollins C, Martin C. Development and psychometric properties of the Birth Satisfaction Scale-Revised (BSS-R). Midwifery [Internet]. 2014 [consultado 31 may 2016]; 30(6): 610-619. Disponible en: http://dx.doi.org/10.1016/j.midw.2013.10.006

49. Leahy P, McCarthy G. Maternal parental self-efficacy in the postpartum period. Midwifery. [Internet]. 2011 [consultado 31 may 2016]; 27(6): 802-10. Disponible en: doi:10.1016/j.midw.2010.07.008

50. Johantgen M, Fountain L, Zangaro G, Newhouse R, Stanik J, White K. Comparison of labor and delivery provided by certified nurse-midwives and physicians: a systematic review, 1990 to 2008. Women's Health Issues [Internet]. 2012 [consultado 02 jun 2016]; 27(1): 73-81. Disponible en: doi:10.1016/j. whi.2011.06.005

51. República de Colombia. Ministerio de la protección Social. Fondo de Población de Naciones Unidas. Modelo de Atención Integral en Salud para Víctimas de Violencia Sexual. Enero 26 de 2011. Bogotá: UNFPA, 2011.

52. Dos Reis MJ, Baena de Moraes MH, Higa R, Bedone AJ. Atención de enfermería a mujeres que sufren 
violencia sexual. Rev. Latino-Am. Enfermagem [Internet]. 2011 [consultado 31 may 2016]; 18(4): [08 pantallas]. Disponible en: http://www.scielo.br/pdf/rlae/v18n4/es_12.pdf

53. República de Colombia. Ministerio de Salud y Protección Social. Colciencias. Instituto Nacional de Cancerología. Sociedad Colombiana de Urología. Guía de práctica clínica para la detección temprana, diagnóstico, tratamiento, seguimiento y rehabilitación del cancer de próstata. 2013. Bogotá: El Ministerio, 2013.

54. Schober M, Gerrish K, McDonell A. Development of a conceptual policiy framework for advanced practice nursing: an ethnographic study. Journal of Advanced Nursing [Internet]. 2016 [consultado 13 may 2016]; 72(6): 1313-1324. Disponible en: doi: 10.1111/jan.12915

55. Ryley N, Middleton C. Framework for advanced nursing, midwifery and allied health professional practice in Wales: the implementation process. Journal of Nursing Management [Internet]. 2016 [consultado 13 may 2016]; 24(1): E70-E76. Disponible en: DOI: 10.1111/jonm.12291 